Lexique vivant de la révolution et de la guerre en Syrie

\title{
Azadî, modulations, \\ mutations et projection \\ des engagements kurdes \\ en Syrie et dans le monde
}

Boris James ${ }^{1}$

${ }^{1}$ Université Paul Valéry Montpellier 3

Published on: Jan 25, 2022

DOI: 10.21428/3633fae9.478d412e

License: Creative Commons Attribution 4.0 International License (CC-BY 4.0). 
Le terme azadî, qui veut dire « liberté », appartient à un très large spectre linguistique indo-iranien qui se déploie sur une aire géographique allant de l'Asie du Sud-Est aux Balkans. Son usage a une histoire et une sociologie extrêmement complexes comme en atteste la profusion de ses occurrences dans le monde indo-pakistanais. Il ne nous appartient pas ici d'explorer ce panorama bigarré, bien que des liens parfois étonnants puissent surgir entre des espaces politiques et culturels divers et distincts dans le cadre mondialisé régi par l’internet.

En Syrie, azadî est devenu l'un des slogans de la révolution de 2011. Pourtant, pour les Kurdes comme pour les arabes syriens, ce terme était jusque là exclusivement associé au combat politique des Kurdes. Depuis le début du $\mathrm{xx}^{\mathrm{e}}$ siècle, il occupe une place centrale dans l'imaginaire politique de ces derniers. Dans la sphère transfrontalière kurde (Syrie, Irak, Turquie, Iran et Europe), il constelle littéralement, aux côtés des termes « serbestî » (synonyme) et « rizgarî » (plutôt traduit par « libération), les discours écrits et oraux des acteurs politiques et culturels. On ne compte plus les strophes de poèmes, les refrains de chansons comme les slogans de manifestations, les titres d'ouvrages, d'organes de presse écrite et audiovisuelle, les noms d'associations littéraires, d'organisations politiques et militaires qui comportent le terme azadî. Plutôt que de faire une recension complète de ces occurrences dans un milieu kurde élargi, nous tentons ici de retracer ses usages passés et présents dans le contexte syrien et surtout d'étudier les implications de la diffusion de ce mot dans le cadre de la révolte de 2011 et du conflit qui a suivi jusqu'à nos jours.

Ce travail exploratoire est fondé sur l'étude du matériau issu de la fréquentation durant une vingtaine d'années des milieux kurdes syriens, d'une quinzaine d'entretiens précis avec des personnalités et activistes syriens kurdes et arabes autour de la révolte de 2011, et sur l'analyse du contenu des médias sociaux (Facebook, twitter, etc.) et des plateformes de téléchargements de vidéo (YouTube, Vimeo) relatifs à ces évènements.

Il s'agit surtout ici de replacer l'usage du mot azadî dans une histoire de la présence kurde en Syrie, afin d'interroger les dynamiques de visibilisation et de dissimulation de celle-ci sur la longue durée. Le focus sur ce terme permet en partie d'étudier la relation des individus et des mouvements kurdes avec la construction nationale syrienne depuis 1946. Observer ce fait de langue et son contexte donne donc à voir non seulement les revendications et l'imaginaire proprement kurdes auquel il renvoie, mais également la projection de cette symbolique vers des espaces non-kurdophones. C'est pourquoi on se penchera ici sur les points de convergence avec les autres 
oppositions syriennes, notamment arabe que l'emploi du terme azadî implique. En tant qu'emblème d'une certaine kurdité, il est évidemment révélateur des modalités de l'engagement des Kurdes dans la révolte syrienne de 2011, mais également de la part que ces mêmes activistes kurdes ont prise dans la maturation et la genèse de cette révolte sur plusieurs décennies.

Après 2013, les années de détérioration de la situation politique et militaire en Syrie ont vu la relative déconnexion du terme azadî des aspirations transversales de la révolution syrienne. Le mot se retrouve plus nettement attaché au projet politique porté par la mouvance hégémonique du Parti des Travailleurs du Kurdistan (PKK). Le potentiel de séduction de ce projet a donné au symbole azadî une postérité renouvelée à l'international qu'il convient d'étudier. L'itinéraire du terme azadî constitue ici un fil rouge révélateur de la teneur des engagements et des structurations politiques au sein de la scène kurde, structurations dont le procès est loin d'être arrivé à son terme.

\section{Du comité Azadî à Cegerxwîn, les premiers chantres de la liberté}

De manière assez surprenante, le terme azadîn'apparaît jamais en tant que substantif dans les premiers dictionnaires ou lexiques bilingues kurdes entre la fin du xviiie siècle et le début $d u x^{e}$ siècle $\frac{1}{}$. On peut supposer ainsi que le concept avait un usage restreint et très éloigné de la teneur politique qu'on lui attribue de nos jours. L'émergence de la « question kurde » née du démantèlement de l'Empire ottoman et de la séparation des zones de peuplement kurde au sortir de la $\mathrm{I}^{\mathrm{e}}$ Guerre mondiale a certainement contribué au rayonnement de ce terme qui renvoyait alors essentiellement à la lutte d'émancipation nationale d'un peuple soumis aux jougs turc, iranien et occidental.

La première association nationaliste kurde, le comité Azadî, fondé en 1923 à Erzorum en Turquie, eut un rôle déterminant dans le soutien au soulèvement de Sheykh Said en 1925느. Composé de militaires transfuges de l'armée ottomane, d'intellectuels kurdistes, de chefs de tribus et d'aristocrates, il opérait clandestinement dans l'est du territoire de la République de Turquie en formation. En exil, le pendant de cette organisation s'appelait la ligue Xoybûn (« être soi-même ») et déployait une intense activité culturelle et diplomatique auprès des grandes puissances, notamment les autorités mandataires. En 1945, le Xoybûn est remplacé par l'association Yekbûn û Azadî (Unité et liberté) $\underline{3}$.

Les milieux intellectuels kurdistes, souvent composés d'exilés de Turquie liés à ces associations, qui publiaient des revues culturelles à Beyrouth et Damas (Hawar, Ronahî 
Les milieux intellectuels kurdistes, souvent composés d'exilés de Turquie liés à ces associations, qui publiaient des revues culturelles à Beyrouth et Damas (Hawar, Ronahî et Roja $N \hat{\text { û }}$ sous le mandat français, ont fortement concouru à la centralité du terme dans l'imaginaire politique kurde, en le liant de manière intrinsèque au projet national. Un des animateurs les plus célèbres de ces revues fut Şexmûs Hesen, alias Cegerxwîn, un poète et activiste de la région de Mardin exilé à Amûda en Syrie. Il avait participé à la révolte de 1925 en Turquie et rejoignit le Xoybûn en 1927, alors qu'il s'installait dans la Djéziré syrienne. En 1944, Cegerxwîn créa la Civata azadî û yekîtiya kurd (association de la liberté et de l'union kurde) et en 1957 le parti Azadî qui fusionna rapidement avec le Parti démocratique kurde en Syrie (PDKS), le seul parti kurde de Syrie jusque dans les années 1970ํ․ Les conceptions politiques de Cegerxwîn ont littéralement infusé ses poèmes que ce soit dans les nombreux recueils ou dans les publications régulières de l'entre-deux-guerres citées plus haut. Bien que ses premières productions n'élèvent pas encore les concepts abstraits de « liberté » et de « libération » au rang de principe cardinal $\underline{5}$, dès après la Seconde Guerre mondiale ces notions conçues comme revendication politique sont sans cesse promues de manière manifeste ou implicite par le poète, tout en prenant une coloration nationaliste grandiloquente et clairement marxiste-léniniste.

Le deuxième recueil de Cegerxwîn compilé en 1954 porte le titre de Sewra Azadî, « la révolution de la liberté ». Ici le terme arabe «sewra » qu'on n’utilise plus de nos jours en kurde - on lui préfère le mot « şoreş » - marque le tournant esthétique soviétisant et marxisant de Cegerxwîn. En effet, reprenant le thème de l'esclavage du peuple kurde dans le poème Kurdistan ne kolistan e (Le Kurdistan n'est pas un « esclavistan »), l'auteur met en avant la libération du peuple (ouvriers et paysans) de l'oppression des aghas et des begs $\underline{6}$, ces autorités traditionnelles honnies par les communistes. Le recueil est traversé par la trahison des notables et la fascination pour l'irrépressible puissance de l’URSS. Le poème éponyme du recueil se conclut ainsi : « Bimrî zordestî bijî azadî (Que meure la tyrannie, que vive la liberté) / azadî perest hemî bûne yek (amoureux de la liberté unissez-vous !) / [...] sewra me ya xurt vî şerê xwîndar (notre révolution puissante, cette guerre sanglante) / xistin destên me tev rejîm û dar (nous fera mettre à bas tout régime et tout arbre) / bijî proletar, bijî Sitalîn (Vive les prolétaires ! Vive Staline !)» $\underline{7}$. 


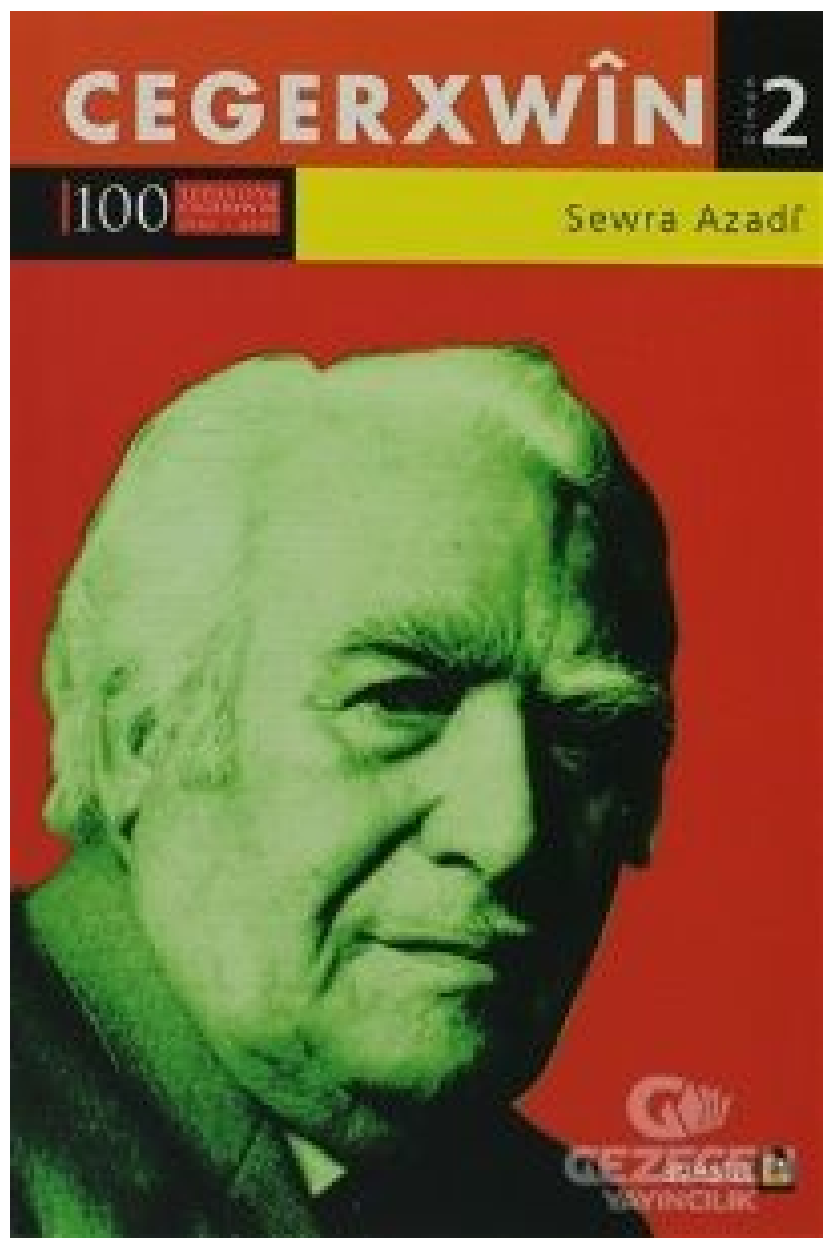

Couverture du recueil de poèmes de Cegerxwîn Sewra Azadî (La révolution de la liberté). Editions Avesta, 2004.

La position de Cegerxwîn vers 1950 est emblématique de l'activisme kurdiste de l'époque. Il est nationaliste, pankurdiste et marxiste, et porte ses revendications en priorité contre les menaces turque et iranienne (donc pas encore arabe) avec une multitude de nuances. Un seul point commun se dégage : la foi dans l'engagement et la capacité du bloc soviétique à libérer le « peuple kurde ». Cette coloration a imprimé durablement le terme azadî asséné comme un mantra par les activistes.

Il faut attendre la fin des années 1950 et le début des années 1960 pour qu'un double virage s'opère. D'une part, les États arabes se mettent à produire un discours nationaliste arabe intransigeant (invisibilisation de la langue et de la culture kurde dans toutes les sphères, y compris le discours historique scolaire) et à mettre en place des politiques discriminatoires concrètes envers les populations kurdes (arabisation des toponymes, installation de familles arabes, regroupement dans des villes collectives, exclusion de la nationalité syrienne, etc.) $\underline{8}$. Ainsi, l’inadéquation de la 
construction nationale syrienne moderne avec la présence kurde, de même que la nonadhésion des Kurdes au « contrat social » mis en place dans le pays, ne date aucunement de l'avènement d'un gouvernement baasiste en 1963 bien que celui-ci ait contribué à sa manière à cette disjonction. Aussi, sur ces bases historiques, les relations des Kurdes à l’État-nation syrien étaient quasiment structurellement antagoniques; ce qui ne manque pas d'être aujourd'hui encore l'objet d'un malentendu tenace.

D'autre part, l'URSS, peut-être du fait même de son soutien aux régimes arabes, n’apparaît plus en 1960 comme la seule puissance tutélaire supportant les espoirs de libération et de liberté des Kurdes. En conséquence, l'activisme kurde s'autonomise des partis communistes locaux et s'ancre plus nettement dans le nationalisme en critiquant, voire en rejetant le cadre des États-nations arabes. Les possibilités de convergence politique avec les gauches arabes, notamment syriennes, furent mises à mal de manière persistante par cette phase historique $\underline{9}$. Ces deux revirements sont vraisemblablement à l'origine de la création du PDKS évoqué plus haut. Pour autant, la militance kurde maintenait son orientation socialisante en Syrie comme ailleurs. À la fin des années 1970, dans la diaspora naissante, l'une des revues les plus lues notamment dans les milieux étudiants portait le nom Azadî et un projet éditorial simple : «siyaset, marksizm, çand » (politique, marxisme et culture) $\underline{10}$. La revue Azadî se dit « revue kurde marxiste » et fait sien l'aphorisme de Ho Chi Minh : ji azadî û serxwebun birûmettir tiştek nîn e (il n’y a rien de plus précieux que la liberté et l'indépendance). En bref, le mot azadî impliquait encore à la fois l'affirmation d'une culture différenciée, la libération nationale et la révolution sociale quels que soient leurs sens, leurs moyens et leurs modalités. Jusque dans les années 1980, la très grande majorité des organisations kurdes gardaient cet ADN avec des nuances diverses et complexes. Parmi elles, le Parti des Travailleurs du Kurdistan (Partiya Karkarên Kurdistan, PKK), mouvement militant et de guérilla venu de Turquie et installé en Syrie et au Liban au début des années 1980, fait un usage pléthorique du terme azadi $1 \underline{1}$. Les slogans affichés, le contenu et les titres des publications du mouvement, les acronymes des sous-organisations usent de manière parfois vague du mot tout en renvoyant à une esthétique léniniste et internationaliste.

Pour autant, en Syrie où il était installé, la « liberté » (azadî) prônée par le PKK ne concernait en rien la possibilité de faire usage de sa langue maternelle dans l'espace public ou d'affirmer une quelconque différence culturelle ou ethnique battant en brèche le nationalisme arabe, de réclamer des avancées démocratiques ou d'établir des formes d'autogouvernement à un niveau local. En effet, pour des raisons tactiques, 
entre les années 1980 et 2000, le PKK et son leader Abdullah Ocalan, alias Apo, ont orienté leur activité militante et militaire depuis la Syrie contre l'État turc, permettant de manière indirecte au régime de Hafez al-Assad de neutraliser la militance et les revendications kurdes locales $\underline{12}$. Néanmoins, ce choix tactique ne réduisait pas à néant l'autonomie de l'organisation ni l'idée que ses militants pouvaient se faire en privé de la nécessité de l'émancipation des Kurdes de Syrie.

À la fin des années 1990, le régime syrien se détourne en partie du PKK et fait expulser Apo sous la pression de la Turquie. En 1998, le leader kurde se retrouve dans les geôles turques et le PKK de Syrie change son nom pour le Parti de l'Union Démocratique (Partiya Yekîtiya Demokrat, PYD) devenant une branche régionale de l'organisation globale. En dépit d'une répression bien réelle de la part du régime et de la création d'un micro-parti d'opposition (Rêkeftin) par une poignée de militants, le $\mathrm{PYD} / \mathrm{PKK}$ n'opère pas le virage qui l'aurait amené à affirmer des revendications concrètes et locales sur la scène syrienne. Le mouvement maintient le cap d'une coopération ambiguë avec le régime. Ce positionnement flottant pouvait alors se retrouvait dans un usage lénifiant du concept de liberté. Le mot azadî continuait à résonner pour s'opposer de manière vague à l'oppression du peuple kurde ou pour critiquer sa répression par l'État turc, très peu pour défendre les militants pourchassés par le régime syrien. Il servait aussi à clamer l'indépassable centralité d'Apo. Dans les années 2000, les célébrations du New Roz, le jour de l' An kurde, en Syrie sont l'occasion de réclamer haut et fort la libération du leader ; entre autres exemples, en 2007 un slogan inscrit sur le fronton d'une scène recouvert d'un portrait « ensoleillé » d'Apo affirme : «La santé du chef, c'est notre santé ; la liberté du chef,

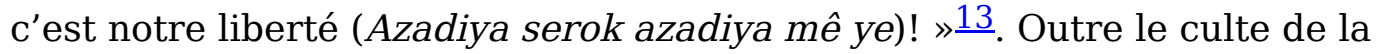
personnalité, cette réduction de l'idée de liberté à la libération du leader visait vraisemblablement à assurer le régime des Assad de l'innocuité des activités du PYD/PKK en Syrie, et du maintien de canaux de communication ou d'une possible coopération avec celui-ci. 


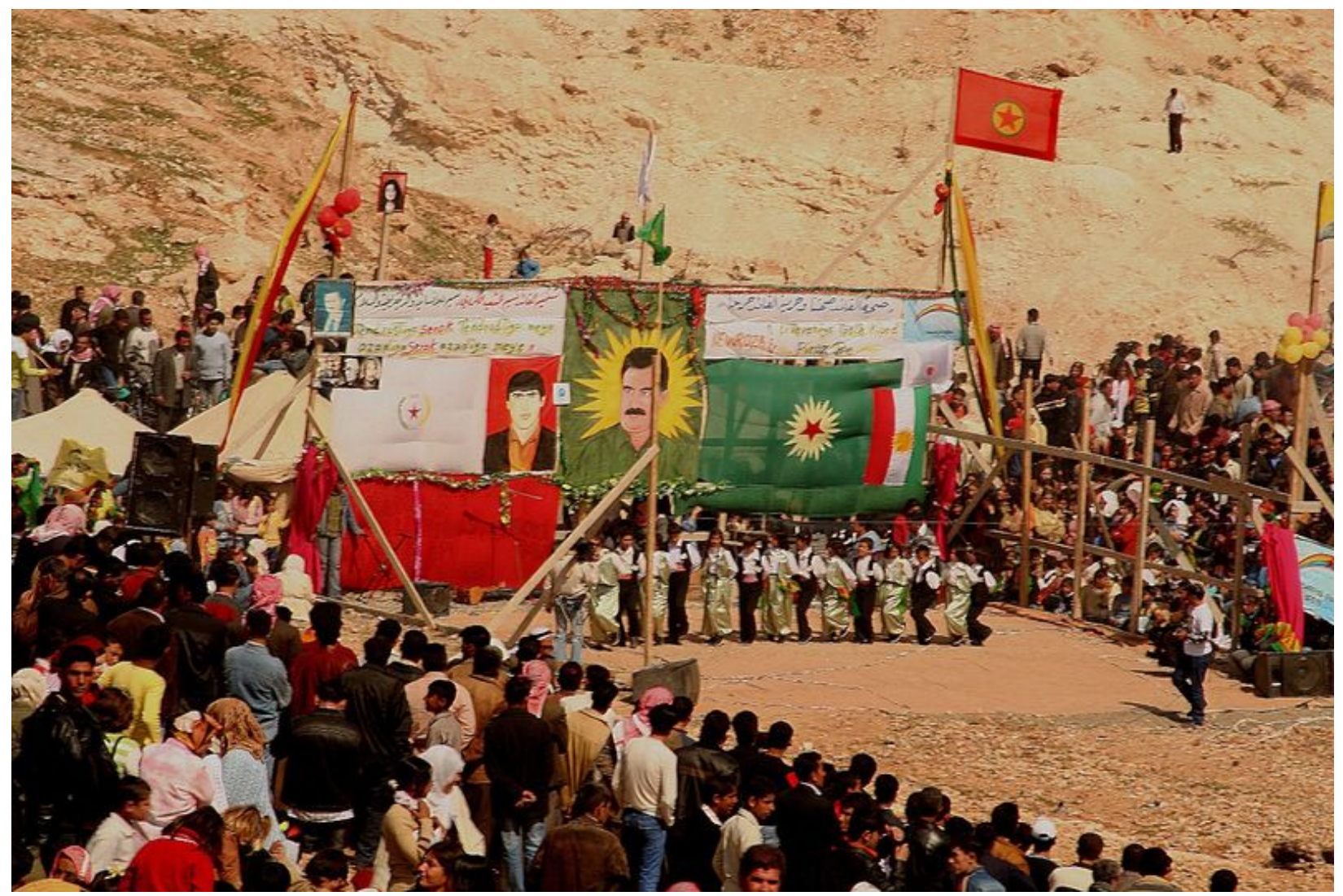

Photo du rassemblement de New Roz, jour de I'An kurde, à Kobane ('Ayn al-'Arab) en mars 2007. Photographie de Jean-François Barthale.

\section{La révolte kurde de 2004 : un avant-goût de révolution ?}

L'historien et anthropologue Jordi Tejel a brillamment montré en quoi le début des années 2000 marque la réémergence de la question kurde en Syrie : c'est-à-dire la remise en cause de l'édifice politique autoritaire syrien à travers le prisme kurde. Les facteurs de cette réémergence sont multiples : une courte phase de libéralisation de l'expression politique en Syrie au moment de l'avènement de Bachar al-Assad au pouvoir ; l'affaiblissement du PKK et de sa capacité de neutralisation de la question kurde en raison du rapprochement entre la Syrie et la Turquie ; l'intervention américaine et l'émergence de pouvoirs autonomes kurdes en Irak. Tout ceci contribue à la naissance d'un nouvel activisme kurde qui réinvente les modes protestataires et s'émancipe des vieilles structures partisanes kurdes issues du PKK et du PDKS souvent compromises avec le régime $\underline{14}$. Entre 2000 et 2002 une série de sit-in prennent place, sous l'impulsion du jeune Parti de l'Union kurde (Yekîtî) notamment pour demander l'attribution de la nationalité syrienne aux enfants descendants des Kurdes qui en furent privés en $1962 \underline{15}$. La question kurde syrienne se manifeste fortuitement, mais 
de manière éclatante au cœur du mois de mars 2004. Lors d'un match de football dans la ville à majorité kurde de Qamişlo une rixe entre supporters du club de la ville et ceux du club de Deir Ezzor fait plusieurs morts du côté des premiers en raison des tirs des forces de sécurité syriennes. Cet évènement vite relayé provoque une série de soulèvements quasi-spontanés dans les villes et les quartiers à majorité kurde du pays, Qamişlo, Derîk, 'Amûda, Dummar al-Balad (Zorava), Rukn al-dîn à Damas, Sheykh Maqsûd et Ashrafiyeh à Alep, etc. Nombre de symboles du baasisme et du régime sont détruits (statues de Hafez al-Assad, portraits de son fils, bannières diverses, etc.). La répression de la part des forces de sécurité est impitoyable. Elle cause la mort d'une cinquantaine de personnes et des milliers d'arrestations $\underline{16}$. Lors de ces manifestations, les slogans les plus entendus sont ceux de « Vive le Kurdistan ! » (Bijî Kurdistan), « les martyrs ne meurent pas ! » (şehîd nemire !) ou « par l'âme et le sang nous sommes avec toi, ô martyr ! » (bican bixwîn biterene ya şehîd !). Côtoyant les cris de « New Rûz ! », « Halabja ! », du nom de la ville kurde d'Irak où des milliers de civils furent gazés en 1988, ils contribuent à donner une forte teneur différencialiste, voire nationaliste à ces rassemblements $\underline{17}$. Seule l'expression « Bijî azadî » (vive la liberté !) pouvait renvoyer à une revendication universelle d'émancipation $\underline{18}$. En dépit de l'absence notable sur le devant de la scène des organisations kurdes, ces évènements révèlent l'existence d'une cosmogonie kurde transfrontalière et transhistorique faite d'incidents violents et d'héroïsme opposant les Kurdes de Syrie à l'État central. Azadî est à la fois le cri de colère des Kurdes de Syrie contre l'arbitraire du régime et leur clameur d'euphorie au regard de la situation régionale qui a vu le renforcement des formations politiques kurdes d'Irak. La projection de l'engagement kurde de Syrie se fait donc essentiellement vers la sphère kurde. Néanmoins, cette teneur nationaliste fut nuancée par l'exhibition de banderoles en langue arabe et la tenue de discours par des personnalités consensuelles, notamment Ma‘shûq al-Khaznawî, un sheykh naqshbandi très populaire qui a été assassiné par le régime un an plus tard $\underline{19}$. Nombre de ces figures de premier plan s'exprimaient en arabe littéraire et réclamaient la sanction des responsables du massacre, la mise en place d'une enquête indépendante et l'octroi de libertés aux Kurdes au nom des droits humains ou « la résolution pacifique de la question kurde en Syrie ». Ainsi, un certain nombre d'acteurs essayaient de syrianiser la révolte, de dialoguer avec les éléments favorables à une réforme du régime, tout en construisant des ponts avec l'opposition arabe sensible à un discours sur la démocratie et les droits de l'homme. La tentative n'eut aucune conséquence immédiate, le régime jouant à fond l'antagonisme arabo-kurde en armant certaines tribus arabes locales, par exemple $\underline{20}$. Seules quelques personnalités de la société civile se montrèrent solidaires des victimes $\underline{21}$. Les rues des grandes villes 
syriennes de l'ouest en dehors des quartiers kurdes, restèrent silencieuses. Il nous apparaît cependant que les signaux lancés à cette occasion d'une possible convergence des oppositions syriennes ont porté leurs fruits quelques années plus tard en 2011, lorsque le vent de la révolte souffla plus à l'ouest et plus au sud. L'exaspération face à l'arbitraire du régime, la volonté de s'en prendre à ses symboles étaient les mêmes dans la Djéziré kurde, dans le Horan ainsi que dans la région de Homs. Elles durent faire leur chemin et surtout trouver à s'organiser dans des formations crédibles. C'est notamment ce que Meshaal Temo, un activiste kurde partisan de la jonction des oppositions kurdo-arabes, contribua à construire à partir de 2005 avec la création du Mouvement du futur. En 2006, aux côtés des partis kurdes les plus récents, dont le Partiya Azadiya Kurd li Surye (Le Parti kurde de la liberté en Syrie) créé la même année, il forma le « comité de coordination kurde » (Komîta Tensîqa Kurd), une coalition dont le nom n'est pas sans rappeler celui des « tansîqiyyât » (coordinations) qui furent à la pointe de l'organisation du soulèvement du $2011 \underline{22}$.

\section{Azadî dans la révolte syrienne de 2011 : affirmer une singularité dans l'union?}

Lorsque la révolte de mars 2011 éclate en Syrie, fort loin de Qamişlo, les jeunes Kurdes répondent présents dès le départ. Dans les quartiers mixtes de Damas (à Rukn al-Dîn, Berzeh etc.), par exemple, ils participent massivement aux rassemblements. Les comités de coordination (tansîqiyyât) se forment et organisent à Hassakeh, Derbassiyyeh, 'Amûda, 'Afrîn, manifestation sur manifestation en synchronie et en synergie avec les mouvements de l'ouest du pays $\underline{23}$. Les étudiants kurdes des grandes villes dites arabes sont parmi les plus enthousiastes. Cette participation montrée en exemple par les autres révolutionnaires est d'ailleurs l'occasion de critiquer l'immobilisme des métropoles dites arabes (Alep, Damas). Parallèlement, l'activisme numérique des Kurdes manifestant leur ralliement à la révolution sur internet est particulièrement assidu et foisonnant $\underline{24}$. Comme les autres jeunes activistes syriens, ils documentent les manifestations, peaufinent leur discours et rendent public publicisent leur opposition commune au régime. Contrairement au soulèvement de 2004, les signaux envoyés ont dès le départ vocation à produire du lien entre Kurdes et Arabes. Le drapeau kurde se trouve apposé au drapeau vert et rouge de la révolution syrienne $\underline{25}$. Il s'agit de présenter ce drapeau, non comme une revendication de séparation, voire de séparatisme, mais comme le « symbole de la présence kurde et de son inclusion dans la révolte et le tissu social syrien » $\underline{26}$. Les slogans de fraternité kurdo-arabe sont scandés et inscrits sur les banderoles. «Un, un, un, le peuple syrien est un !» ; « Ni arabe, ni kurde, nous voulons l'unité nationale ! » $\underline{27}$; « Kurdes, Arabes, 
on ne nous humilie pas !»; « kurde et arabe, [notre révolte est] pacifique ! » $\underline{28}$. L'activiste Meshaal Temo prend la parole à plusieurs reprises lors de discours vibrants à 'Amûda et Qamişlo pour indiquer, tout en signalant son respect pour le mouvement national kurde, sa pleine participation et celle de ses compagnons à la « révolution syrienne ». Cette prise de position lui coûte la vie en octobre 2011으. Toutefois, la formule prend. Les manifestants des villes arabes et kurdes se répondent et se saluent d'un vendredi à l'autre $\underline{30}$, parfois dans la langue respective de leurs interlocuteurs à l'instar des slogans échangés entre 'Amûda, Kfar Nabl et Zabadânî̉․ On assiste à l'échange de signes de part et d'autre d'une frontière symbolique arabo-kurde que l'on veut de plus en plus labile. De la même manière, la scène kurde transnationale transforme la révolution syrienne en cause kurde. La chanteuse kurde de Syrie exilée au Kurdistan de Turquie, Mizgîn Taher, se joint à la star kurde de Turquie, Nizamettîn Arîç, pour une chanson (Eşa Rojava, Douleur du Rojava) évoquant le Sheykh Ma'shûq al-Khaznawî et Meshaal Temo. Le clip se termine par la scansion de azadî par les manifestants de la révolution syrienne $\underline{32}$. Le chanteur kurde de Turquie Şivan Perwer, compose une chanson en arabe intitulée « El Selam wel Hurriye » (Aştî û azadî) en hommage au jeune Hamza al-Khatib $\underline{33}$, jeune manifestant de Deraa, torturé à mort par les services de sécurité en avril 2011. Dans cette même chanson, il salue son camarade Samih Shqayr qui dans les années 1980 avait écrit une chanson intitulée, «j'ai un ami du Kurdistan et son nom est Şivan » $\underline{34}$, ce qui lui « avait causé beaucoup de tort » $\underline{35}$. Quelques mois plus tard, les deux chanteurs qui ne se connaissaient que de loin en loin participent à Berlin, à un concert de soutien à la révolution syrienne, sous une banderole sur laquelle est inscrit « Azadî, Freiheit » $\underline{36}$. 


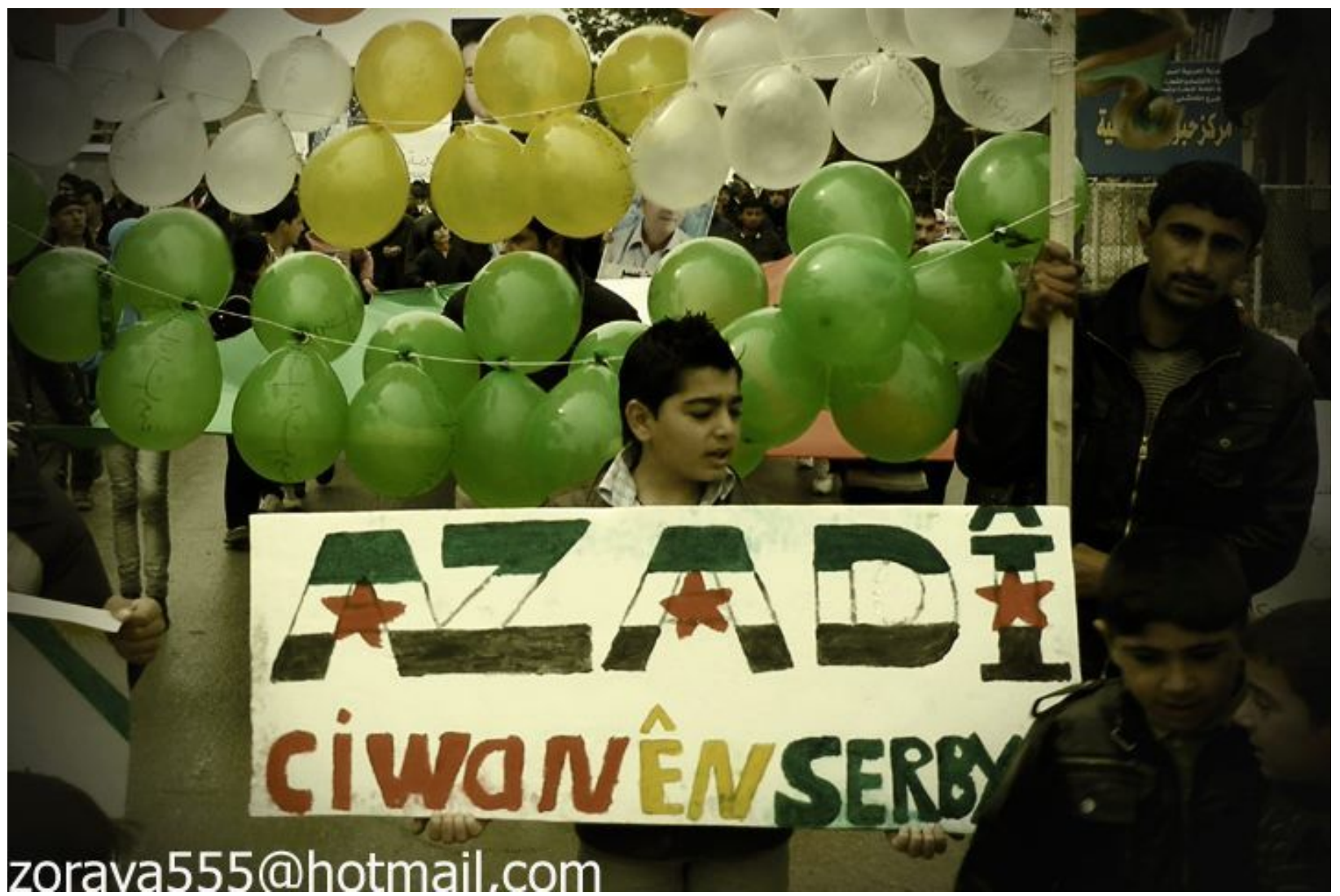

Enfant portant une pancarte sur laquelle est inscrit « azadî » aux couleurs de la révolution syrienne.

https://www.facebook.com/LensKurdishYoung/photos/107042756109408

Le terme azadî représente en effet la transversalité de la révolution syrienne. En 2011, il est d'abord inscrit sur des pancartes dans les villes de la Djéziré et scandé par les habitants kurdes des quartiers mixtes des grandes villes comme gage de leur participation à une lutte plurielle. Notons qu'entre mars 2004 et mars 2011, « bijî azadî » (vive la liberté !) a fait place à azadî tout court. Cet énoncé simplifié, se fait plus cinglant et accentue l'analogie avec la scansion de 《hurriye ») par les manifestants syriens des premiers rassemblements à Deraa et à Damas. Il permet de souligner l'engagement pour une cause commune. C'est ce qui amène la coordination en charge de nommer les manifestations du vendredi à intituler le rassemblement du 20 mai 2011 «Vendredi azadî » $\underline{37}$. Ce choix qui fut le fait, on peut le supposer, d'une majorité d'activistes arabes, a été perçu très positivement par les milieux kurdes, qui signifièrent leur gratitude lors de ce rassemblement $\underline{38}$. 


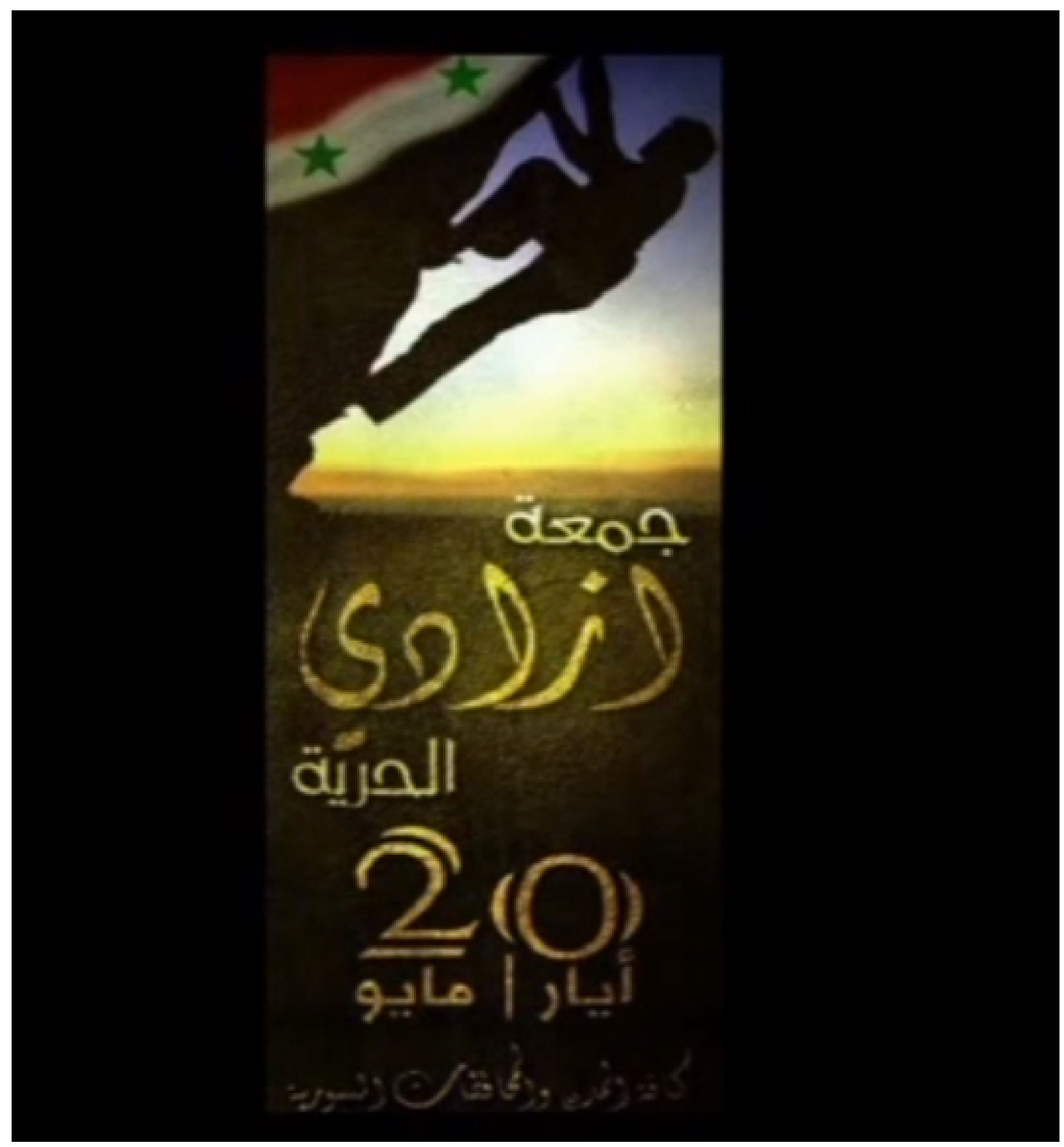

Affiche appelant à la manifestation du Vendredi azadî. Anonyme.

Azadî n'est pas la simple traduction du mot arabe hurriye (liberté) scandé lors des évènements de mars 2011. Il porte une multitude de significations et implique autant de positionnements dont la juxtaposition suggère l'ambivalence. Tout d'abord, le choix de cette appellation pour un vendredi de manifestation revient à ouvrir la sphère publique syrienne et arabe à la différence culturelle kurde et revient à annoncer qu'on s'engage dans la révolution pour construire une société plurielle et pluraliste $\underline{39}$. Nos 
interlocuteurs, qu'ils soient kurdes ou non, établissent une équivalence entre le choix d'azadî et l'utilisation dans certains slogans du nom de Sultan Al-Atrash, leader druze révolté contre les Français dans les années 1920, ou encore de celui de Salih al-'Alî, rebelle alaouite de la période mandataire, pour désigner le vendredi de manifestation du 17 juin 2011는. Il est bien question de diversité, mais aussi du potentiel révolutionnaire inscrit dans chacune des composantes de la société syrienne qui sont appelées à rejoindre le soulèvement. C'est aussi l'occasion de rendre hommage à l'activisme des Kurdes, à leur longue lutte contre le régime révélée au grand jour en 2004. Ainsi, un an plus tard, la même coordination maintient sa ligne pluraliste et baptise un des vendredis de la révolution « fidélité au soulèvement kurde [de mars 2004] » $\underline{41}$. Le lien entre 2004 et 2011 est clairement établi et revendiqué.

Du point de vue des Kurdes, l'usage du terme azadî dans un espace largement dominé par la langue arabe, signifie l'acceptation de leur visibilité et de leur singularité. Cette reconnaissance ouvre les perspectives de l'appartenance dans la différence à l'édifice syrien, de la sortie de la subalternité et de la construction d'un « contrat social » égalitaire au moins du point de vue des signes culturels. Clamer azadî dans un vaste espace activiste syrien multilingue a également une vocation pédagogique pour les Kurdes de Syrie. Il s'agit de renseigner sur la présence kurde et d'enseigner le pluralisme aux Syriens qui rallient l'opposition au régime. C'est pourquoi le mot azadî est inscrit sur d'immenses drapeaux syriens à Qamişlo, 'Amûda et ailleurs $\underline{42}$. Le 20 mai 2011, sur le drapeau déployé à 'Amûda, on peut lire «AZADÎI= حرية 》 (azadî = huriyya). Il s'agit d'affirmer l'identité des deux termes, signifiant liberté en kurde et en arabe. L'usage du symbole mathématique atteste la visée pédagogique de cet axiome $\underline{43}$. On retrouve cette connotation éducative lorsqu'une très jeune manifestante de 'Amûda détourne les paroles de la chansonnette 《ألف باء بوباية (Alif ba' bubaya)4ㄴ que les élèves syriens récitent pour apprendre l'alphabet arabe pour y placer azadî. L'objectif est d'« inculquer » les significations d'azadî dans la société. Notons que le dispositif suggère aussi un renversement des modalités du social : une « subalterne » à plusieurs titres, - kurde, femme et mineure -, inculque le concept de liberté à la société tout entière. 


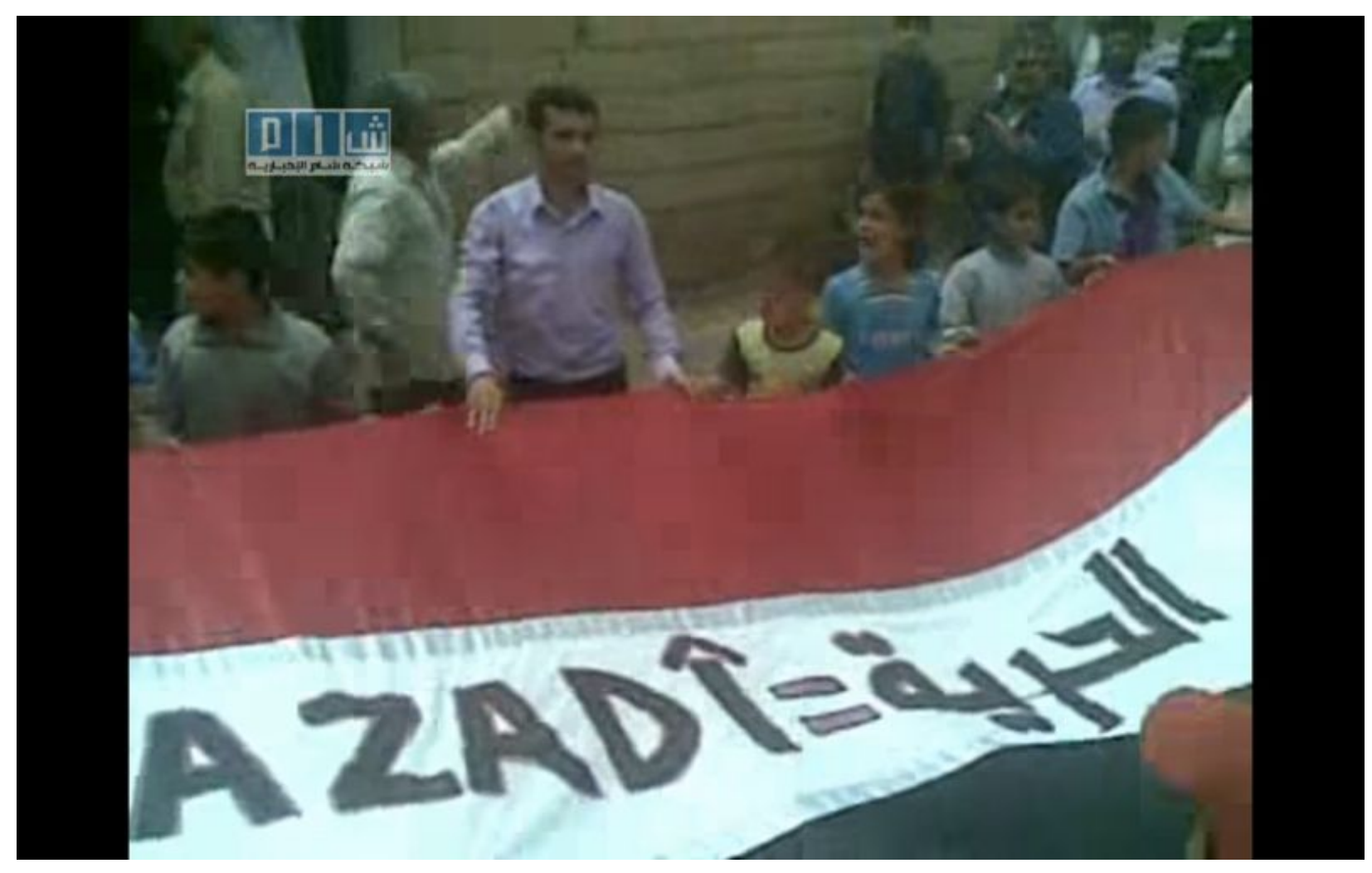




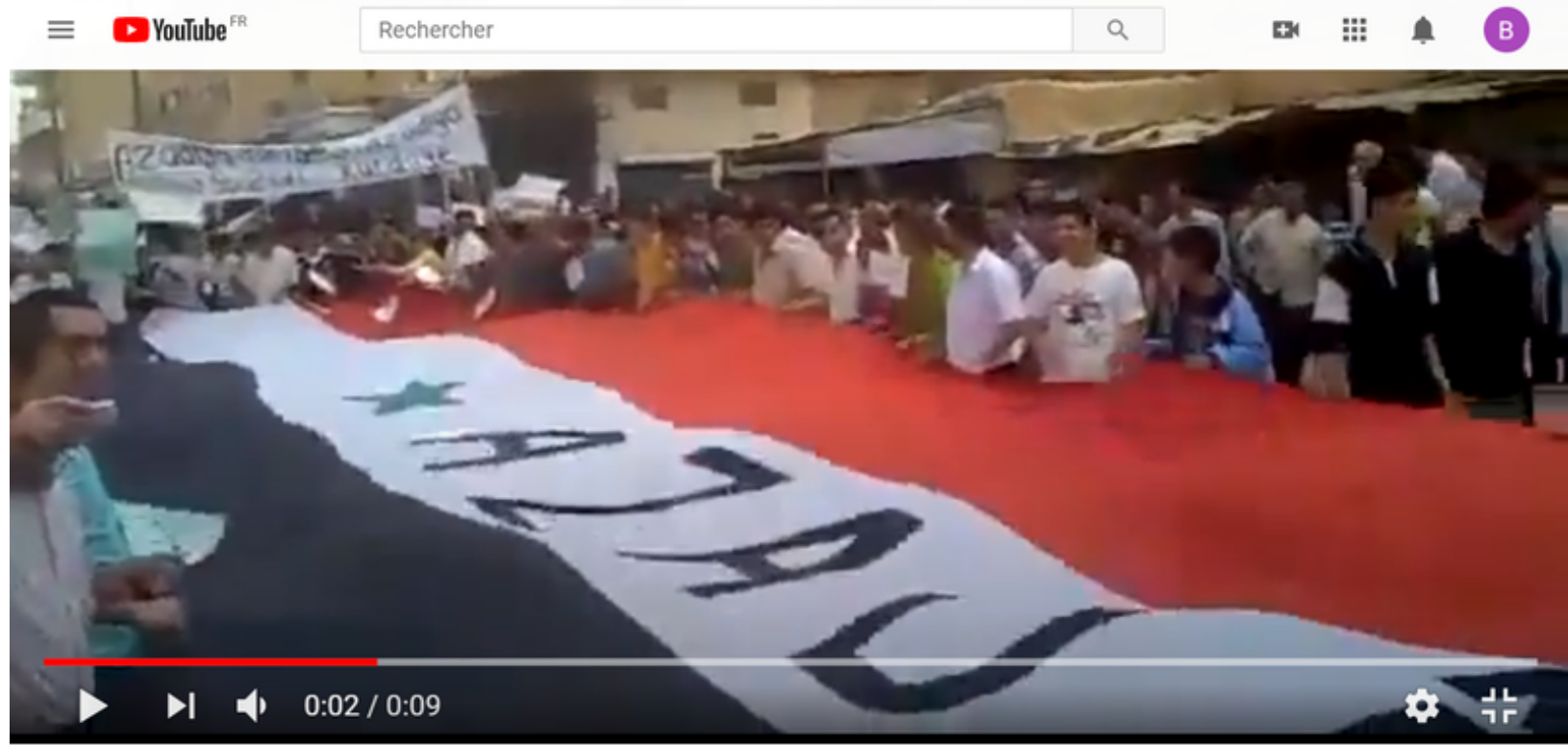

تُورة سوريا - قامشُلو جمعة از ادي 205-011-205

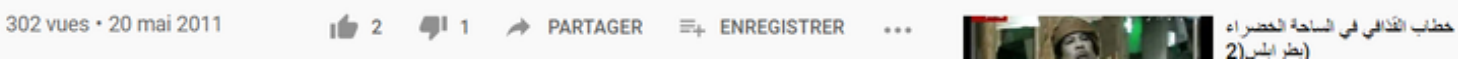

Les drapeaux syriens géants marqués du mot kurde azadîet de son équivalent arabe huriyya à 'Amûda et Qamişlo, jour du Vendredi azadî, le 20 mai 2011.

Captures d'écran de vidéos diffusées sur YouTube.

https://www.youtube.com/watch?

$\underline{v}=\mathrm{dPhR} 9 \mathrm{RT} 5 \mathrm{~d} \mathrm{w} ; \mathrm{https}: / /$ www.youtube.com/watch? $\mathrm{v}=\mathrm{J} \underline{4 \mathrm{P} \times 8 \mathrm{eHBc}}$

Ainsi, l'engagement de certains acteurs kurdes est total, en dépit de nombreuses difficultés. Ces acteurs s'impliquent de manière indépendante au travers de sociabilités kurdo-kurdes ou kurdo-arabes déjà anciennes. Néanmoins, les formations politiques kurdes se montrent également présentes. Le Mouvement du futur de Meshaal Temo intègre le Conseil National Syrien (CNS), l'organisme qui tente de chapeauter la révolution. D'autres formations, la majorité des partis kurdes de Syrie à l'exception du PYD/PKK, constituent le Conseil National Kurde de Syrie (CNKS, ENKS) qui rejoint la révolution sans intégrer totalement le CNS dont ils s'autonomisent d'ailleurs en 2016트. Lorsque la révolte se meut en conflit armé, l'Armée Syrienne Libre, à laquelle on attribue le rôle de défendre et représenter la révolution, intègre une série de brigades ou de bataillons composés de combattants kurdes, environ une dizaine de plus ou moins grande taille, portant les noms évocateurs de «Descendants de Saladin », «Brigade Meshaal Temo » $\underline{46}$ ou « Bataillon Azadî » (كتيبة ازادي) Ce dernier, composé de plusieurs dizaines de combattants, constitue l'un des groupes armés kurdes les plus cohérents. Fondé début 2012 dans la région d'Alep, il affiche un rejet clair et net du régime et des organisations affiliées au PYD/PKK estimant qu'elles collaborent avec le premier. Il met en avant sa kurdité, en exhibant le drapeau kurde, 
et affirme défendre l'amitié entre Arabes et Kurdes sur la base de leur appartenance à l'islam sunnite. Il coopère avec les autres groupes rebelles armés issus de l'Armée syrienne libre, et, depuis 2016, revendique une appartenance à l'Armée Nationale Syrienne qui en émane et manifeste une certaine accointance avec l'islam politique $\underline{48}$. Contrairement à la plupart des partis kurdes de Syrie, dont le Partiya Azadiya Kurd, le bataillon Azadî rejette toute idée d'indépendance ou d'autonomie kurde, n’hésitant pas non plus à se rapprocher ouvertement de la Turquie, tout en clamant sa sympathie pour le Gouvernement Régional du Kurdistan d'Irak $\underline{49}$. Alors que les activistes kurdes qui s'engagèrent dans la phase pacifique de la révolte syrienne critiquent très vivement le rôle de ces organisations militaires, le terme azadî a aussi conféré une coloration kurde au conflit armé syrien.

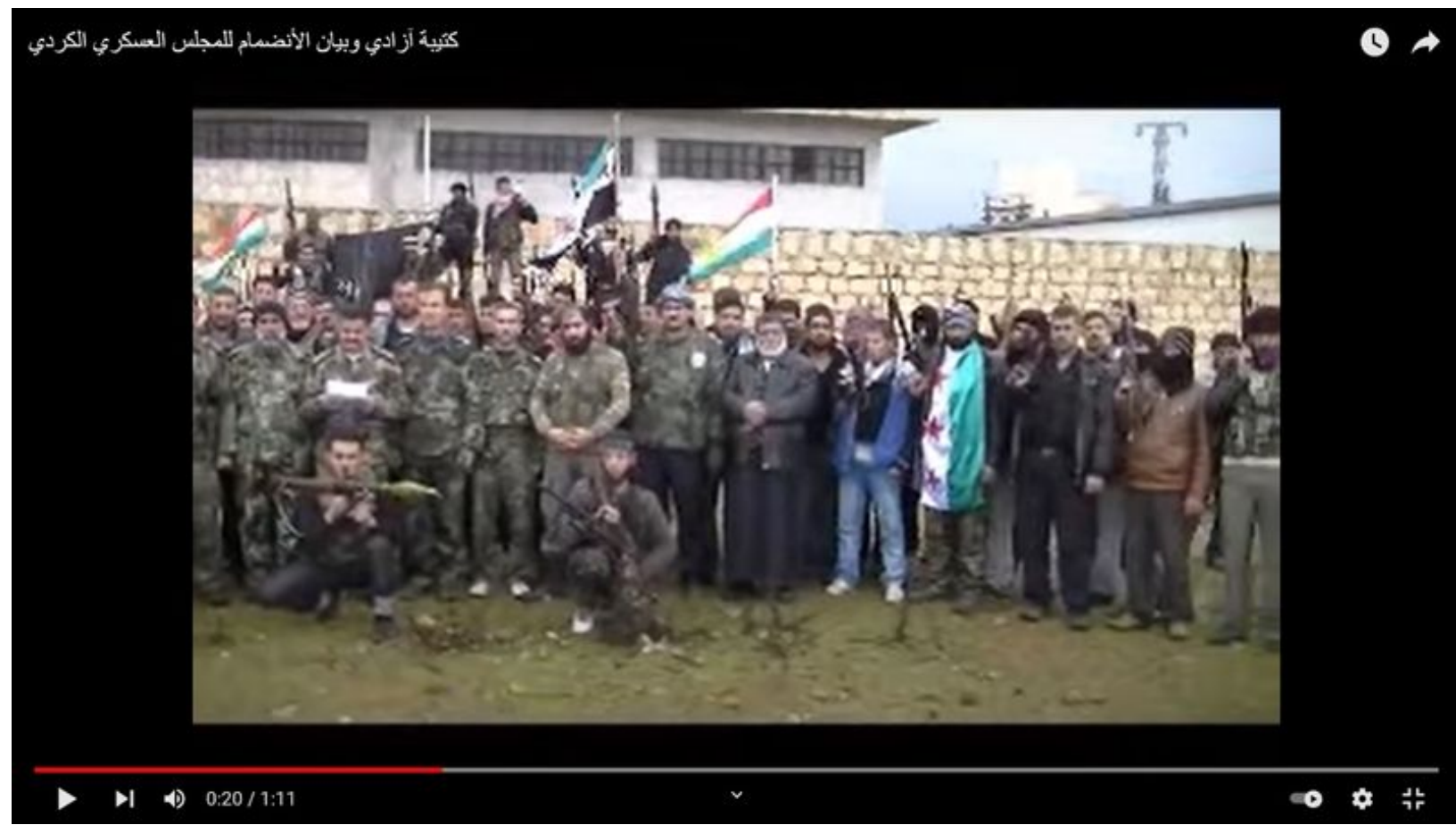

Déclaration d'intégration du Bataillon Azadîau conseil militaire kurde, en 2015. https://www.youtube.com/watch?v=pGtmKghQ5nc

\section{Bien vus, mais « mal-entendus » : la clôture de l'espace kurde en Syrie}

Le mot azadî est devenu le synonyme de l'adhésion complète des Kurdes à la révolution syrienne et symbolisait le refus de toute compromission avec le régime des Assad. Pour autant, aujourd'hui ce fait tend à être occulté par certains observateurs. Pourquoi ? Qu'est-ce qui explique le désengagement réel ou supposé des Kurdes ? Tout d'abord, il est important d'indiquer que la participation ostensible et ostentatoire des 
Kurdes dans la révolution à travers l'usage du slogan azadî était dès l'origine porteuse d'une certaine ambiguïté, voire d'un malentendu autour du sens à donner à ce fait. Si azadî renvoie à la reconnaissance d'une différence, c'est justement sur ce qu'elle implique, sur la place à donner à cette différence dans la construction sociale et politique à venir que réside les malentendus. La reconnaissance et l'affirmation de cette différence ne mèneraient-elles pas à la séparation, voire au séparatisme ? Quid de la rupture avec l'ethos nationaliste arabe du baasisme et quid du rejet de la centralité de l'arabité présente non seulement dans les structures du régime, de l'État, mais également dans la culture politique de la société et des oppositions arabes au régime ? Aucune de ces questions ne trouvait à s'exprimer en 2011, et encore moins trouvait-elle de réponse.

À plusieurs reprises et à demi-mot, ce malaise apparaît dès les manifestations de 2011, qu'il s'agisse de leur déroulement ou de leur couverture par divers médias. D'une part, le nationalisme kurde pouvait refaire surface lors des manifestations ou autour, et d'autre part, apparaissait la gêne, voire le rejet des acteurs arabes vis-à-vis de l'expression de la différence kurde et notamment, vis-à-vis du terme azadî. Çà et là, il est rapporté que dans telle manifestation on a arraché le drapeau kurde des mains d'un manifestant, notamment à Zabadanî en 2012는. Ainsi, en milieu arabe , la plupart des manifestations du vendredi azadî ignorent les références à la question kurde ou à la présence kurde en Syrie $\underline{51}$, alors que, par ailleurs, les références à une arabité centrale et primordiale y sont monnaie courante. Les reportages des télévisions d'opposition d'expression arabe ajoutent souvent le terme arabe huriyye à l'énoncé du nom de ce vendredi spécial. Parfois le terme azadîn'apparaît plus. À la télévision libanaise MTV, Murr TV, du nom d'un député libanais opposé de longue date à la mainmise des Assad sur la région, la couverture des évènements du vendredi azadî ne renvoyait plus que de manière vague à la diversité culturelle en Syrie. Le commentaire indiquait:« entre azadî et Hurriye, on trouve les slogans qui unissent les langues des insurgés syriens, sortis par milliers [dans les rues] en demandant la liberté, la libération des prisonniers et la chute du régime $» \underline{52}$. 


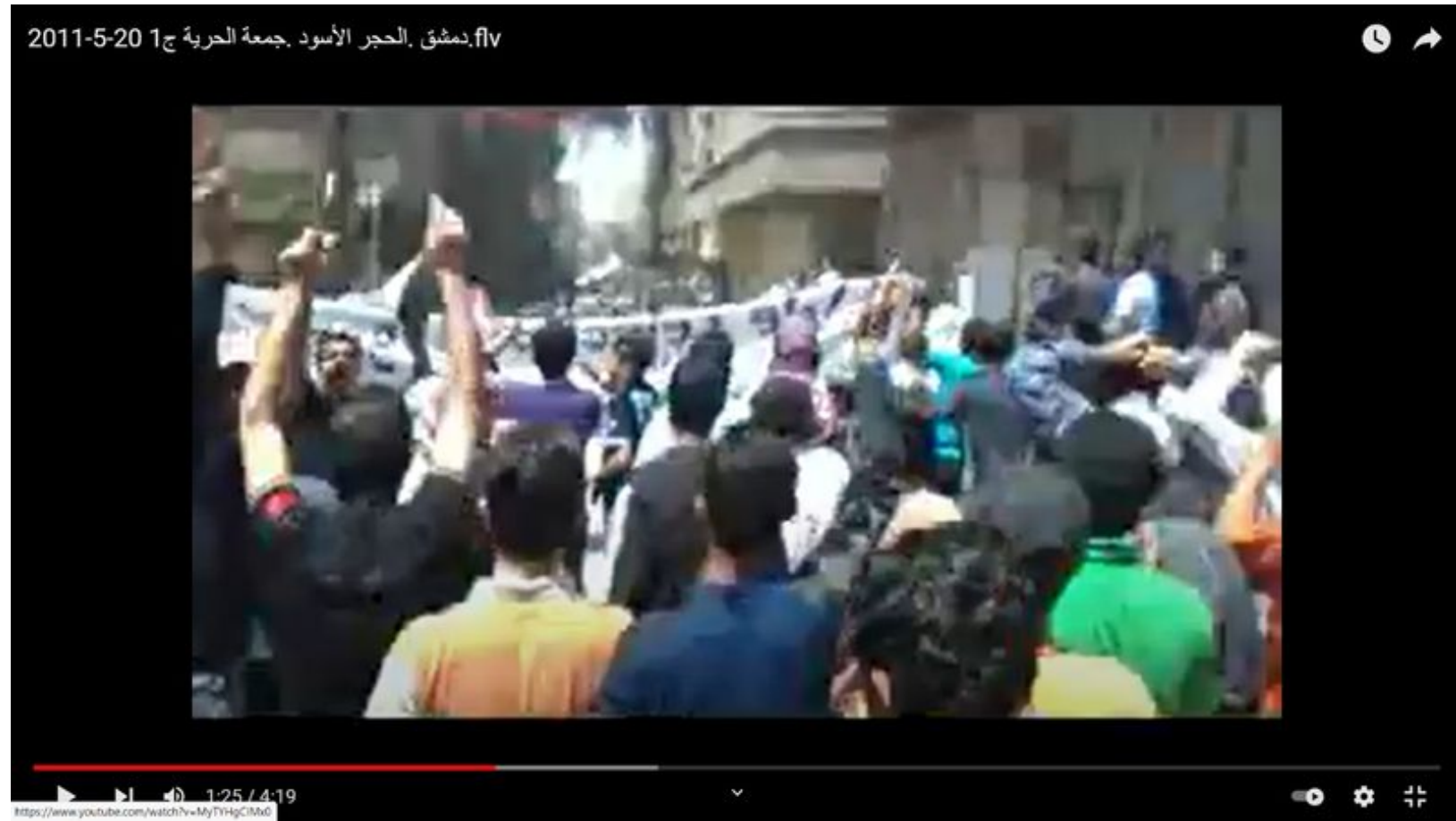

A Hajar al-Aswad le 20/05/2011, il n'est fait aucune mention des Kurdes ou de azadî, lors de la manifestation et dans la vidéo qui en rend compte. https://www.youtube.com/watch?v=2uEsvaRohk0

Il est certain que le nombre important de victimes tombées ce vendredi-là, surtout dans les régions arabes, a largement occulté la portée de cette mise en scène de l'implication symbolique des Kurdes et de leur langue dans la révolution $\underline{53}$. Cependant, la question kurde sous-jacente au choix du nom de cette journée de manifestations est éludée par les acteurs, et l'effet pédagogique recherché de la célébration d'azadî est escamoté par un discours inclusiviste restreint. On peut former l'hypothèse que la peur de la désunion a amené les commentateurs à se replier sur un mode d'expression allusif et euphémisant évacuant les questions qui fâchent.

Du côté des Kurdes, dans la deuxième moitié de l'année 2011, les revendications de fédéralisme et d'autonomie se font de plus en plus pressantes au point d'apparaître sur certaines banderoles et dans les slogans $\underline{54}$. Les inquiétudes par rapport à la satellisation de l'opposition syrienne par la Turquie d'Erdogan se font également ressentir et s'expriment dans les manifestations tenues dans les régions kurdes $\underline{55}$. Les déclarations du président du CNS de l'époque, Burhan Ghalioun, allaient dans le sens de la reconnaissance de l'identité kurde en Syrie, de l'octroi de droits culturels et linguistiques, et d'une certaine décentralisation administrative ; des avancées significatives par rapport au traitement par le régime de la question jusqu'alors. Pour autant, dans les milieux kurdes ces propositions paraissent insuffisantes et 
l'affirmation un peu abrupte de l'homme politique syrien selon laquelle « il n'existe rien de tel qu'un 'Kurdistan de Syrie' » est très mal perçue $\underline{56}$.

On peut situer la rupture vers le milieu de l'année 2012. Ses causes sont multiples et elle se manifeste par plusieurs symptômes. Un exemple de ce retour des Kurdes au différencialisme a été la production fin 2013, par le rappeur Şerîf Omerî, originaire de Qamişlo, de la chanson «Bankin Azadî ! (Clamons la liberté !), dont le contenu et l'esthétique évincent toute possibilité d'inclusion des revendications kurdes dans le cadre de la révolution syrienne, en renvoyant exclusivement à l'imaginaire pankurde évoqué plus haut : la séparation du Kurdistan en quatre morceaux et l'insistance sur la défense de l'identité kurde $\underline{57}$. La chanson «Eşa Rojava » de Mizgîn Taher et Nizamettîn Arîç évoquée plus haut renvoyait à des figures kurdes de la révolution syrienne tout en présentant un panorama kurdo-kurde de la situation de 2012 et en suggérant déjà une disjonction entre territoires arabes et kurdes et donc entre oppositions arabes et kurdes au régime. Quelques années plus tard, si les slogans demandant la chute du régime ou le départ de Bachar al-Assad restent présents dans les manifestations organisées par les premiers compagnons de route de la révolution syrienne, il est surtout question d'affaires kurdes. Ainsi, lors de la commémoration des dix ans du soulèvement kurde du 12 mars 2004, aux côtés du slogan de la révolution en arabe « le peuple veut la chute du régime », on entend, lors d'une manifestation du PDKS sans grande ferveur, en kurde « azadî, fedralî, Bashsharo xwe bide alî » ( « Liberté, fédéralisme, Bashsharo [al-Assad] écarte-toi ») ou « azadî, fedralî seroke mê Barzanî » (« Liberté, fédéralisme, notre chef est Barzani ! »)토. Il est question ici de Masûd Barzanî, célèbre leader kurde d’Irak, fils de Mullah Mustafa Barzanî qui mena la plus longue guérilla kurde de l'histoire (1930-1977). Ceci permet de souligner, d'une part la forte aspiration de certains Kurdes de Syrie à la séparation de l'espace syrien, et d'autre part, l'importance de l'influence historique du mouvement barzaniste sur la scène kurde de Syrie, voire la captivité des mouvements kurdes syriens non-affiliés au PKK, vis-à-vis du Parti Démocratique du Kurdistan d’Irak ; captivité qui est allée en augmentant depuis la fin 2011. 


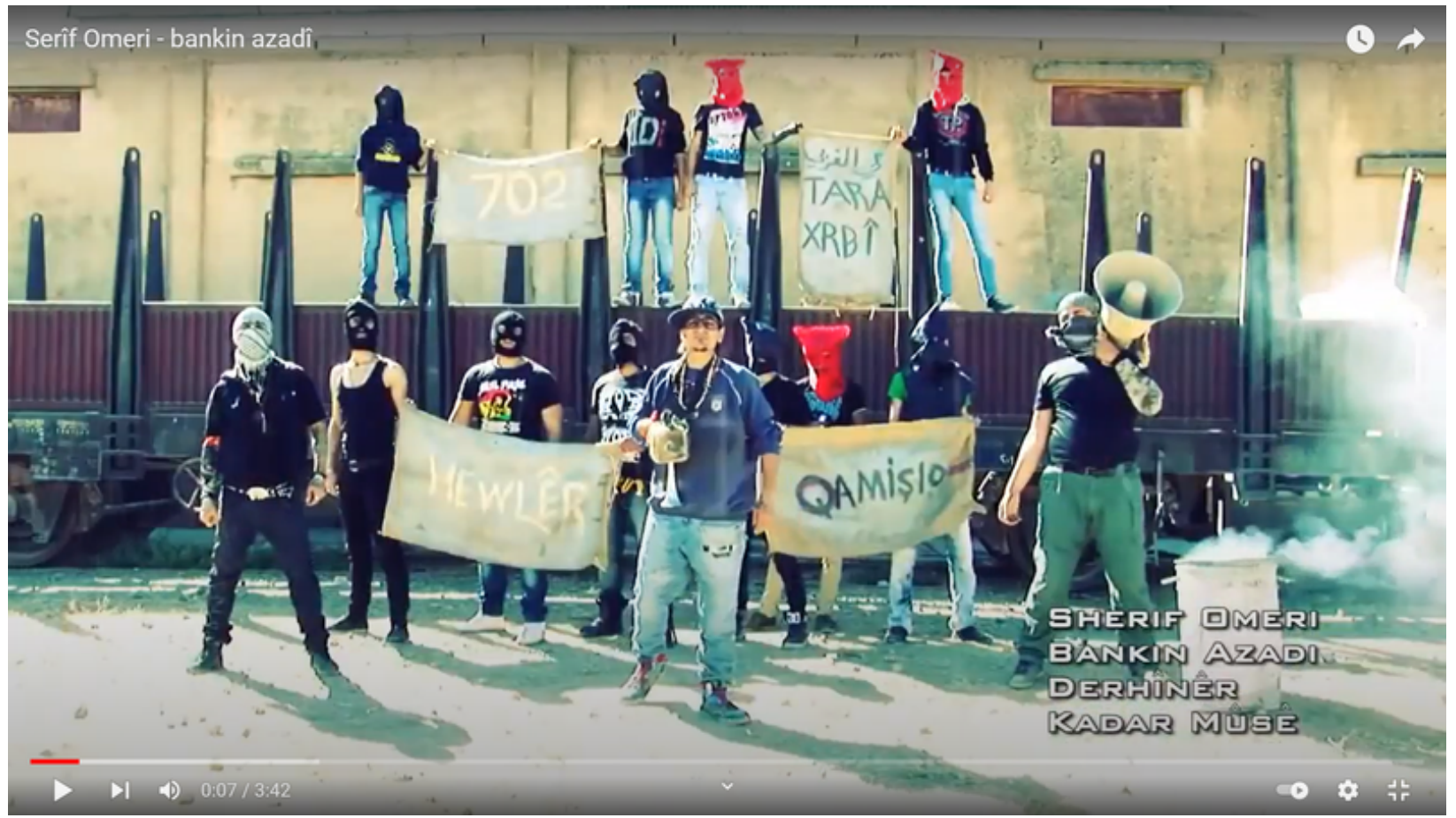

Image du clip de la chanson « Bankin azadî » de Sherif Omeri en 2015. https://www.youtube.com/watch?v=ube_rYhGvdQ

Aux tropismes contradictoires des activistes arabes et kurdes influencés par leurs tendances nationalistes respectives, il convient d'ajouter les conséquences de la montée en puissance des groupes jihadistes arabes ou internationaux. Ces derniers affichent une très forte hostilité envers les activistes kurdes souvent attachés à une militance teintée de sécularisme quelle que soit leur proximité avec le PYD/PKK ou la mouvance barzaniste. Dès 2012, des groupes armés jihadistes sont actifs dans la région de Serekaniye (Ra's al-'Ayn) et de Kobane ('Ayn al-'Arab). La progression de ces groupes culmine dans la conquête d'un territoire immense entre l'Irak et la Syrie par l'Organisation de l'État islamique (Daesh) dans une zone contiguë aux régions kurdes, contribuant en partie à la séparation entre sphères kurdes et arabes et à la mise en place de l’hégémonie locale des organisations armées proches du PYD/PKK.

Les expériences kurde et arabe syrienne se rejoignent néanmoins dans l'exil. Les départs du pays explosent à partir de 2014. L'idée de la migration douloureuse est le premier élément d'interprétation qu'Abdal-Karim Majdal Al-Beik, artiste plasticien kurde syrien exilé à Berlin, me donne pour lire le tableau Memory, confectionnée en 2016 sur lequel sont inscrits, entre autres, les mots «freedom 》, 《رية 》 et 《azadî », ce dernier étant le plus apparent. Il s’agit pour l’auteur d'évoquer ces départs forcés vers l'inconnu et d' « inscrire sur les murs ses désirs les plus intimes. De les ancrer et de s'ancrer dans la pierre au moment de partir ». Il ne souhaite donner « aucune 
dimension politique » à ces inscriptions. Face à mon insistance à vouloir relier ces signes aux évènements de 2011, il m’indique qu'«évidemment, ils peuvent renvoyer à ce moment politique, mais que pour un Kurde, azadî évoque une multitude de révoltes kurdes contre la tyrannie, aussi bien 2011 que 2004 et 2014 ». L'artiste qui souligne son absence d'appartenance partisane reconnaît que le choix du nom azadî pour un des vendredis de la révolution « a été une chose positive. Un moment d'ouverture et d'acceptation de l'autre qui a représenté un espoir fort. Mais, après tout ce qui s'est passé, je me demande encore si l'initiative était sincère ». $\underline{59}$ Pour une certaine frange d'intellectuels kurdes de Syrie azadî est également synonyme de désenchantement, d’un espoir déçu que l'on garde secrètement enfoui en soi. 


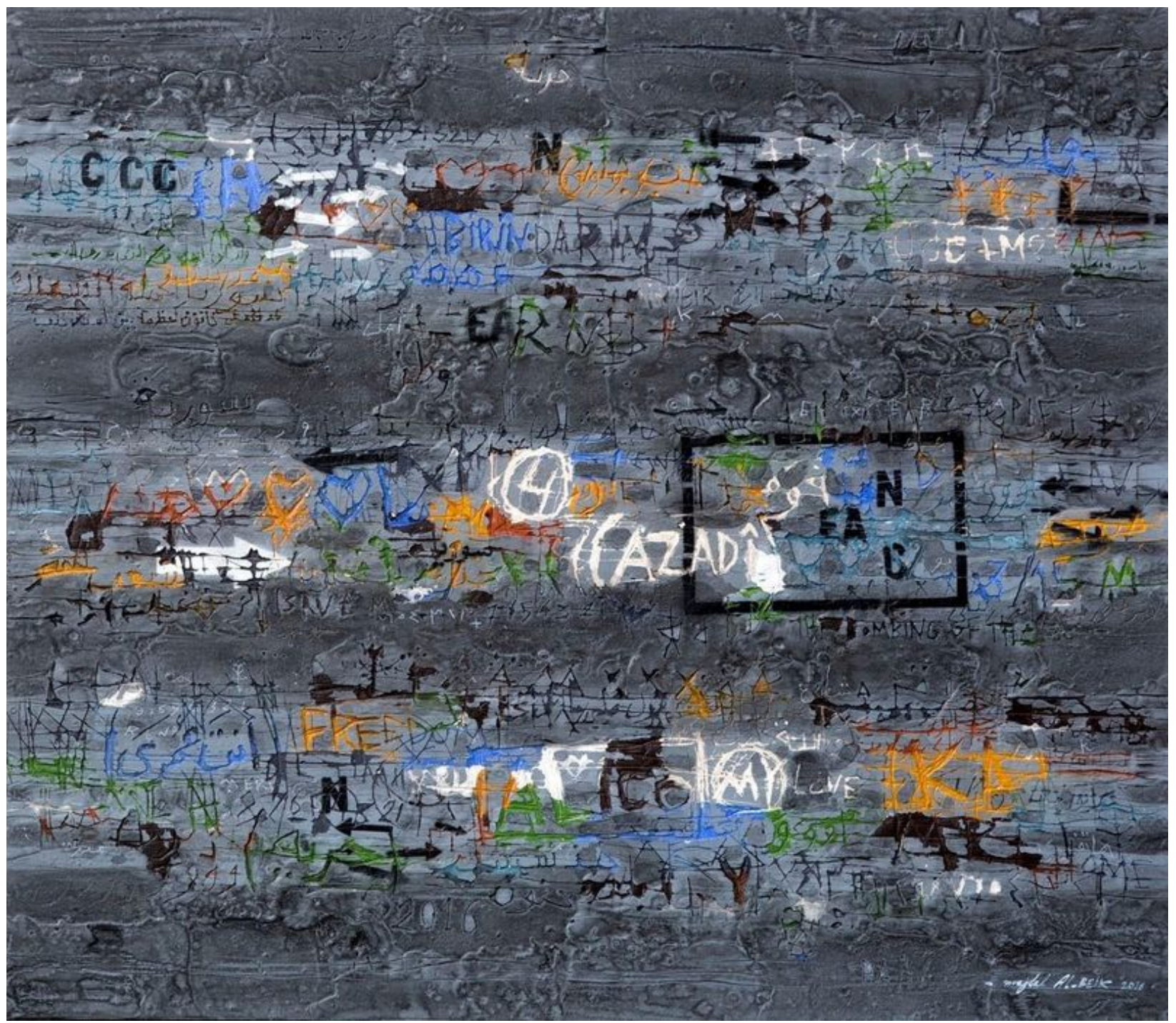

Tableau d'Abdul Karim Majdal Al-Beik, Memory, $130 \mathrm{~cm}$ x 150 cm, 2016, photo de l'artiste.

\section{Du cri de guerre des YPJ au label azadî}

Il semble néanmoins certain que le facteur le plus important du « désengagement » kurde dans la révolution syrienne a été la cogestion de la « crise » par le régime et le PYD/PKK. En effet, les services de sécurité syriens, en connivence directe ou indirecte avec le PYD/PKK, ont déployé dès 2011 une stratégie relativement astucieuse pour traiter la contestation kurde. Tout d'abord, la répression des manifestations dans les zones kurdes a été beaucoup moins frontale que dans les régions arabes. Le nombre de victimes y était, par exemple, très inférieur. Le régime a choisi de cibler une série d'opposants kurdes plutôt que de punir massivement la population comme en 2004 . Par la suite, il a sous-traité la perturbation des manifestations au PYD/PKK qui s'est 
mis a exercé des fonctions de police au niveau local ; ce qui est allé de pair avec l'emploi d'une certaine violence dont l'effet dissuasif ne fut pas négligeable. Cette collaboration avec le régime a culminé avec le retrait partiel des services de sécurité syriens des zones civiles kurdes en 2012. Ils ne se maintenaient plus que dans quelques bases à Qamişlo et Hassakeh. En 2013, le TEV-DEM (Tevgera Civaka Demokratîk, Mouvement de la société démocratique), l'instance englobant les institutions de gouvernance civile de la Fédération de Syrie du Nord (Federaliya Demokratîka Bakûrê Sûriyê), déclarait officiellement l'autonomie du Rojava (Kurdistan de l'Ouest), composé de ses trois régions : la Djéziré, Kobane et Afrîn. Le PYD/PKK a alors interprété ce retrait comme une conquête et comme le point de départ d'une révolution à part entière fondée sur les principes du confédéralisme démocratique édictés par Apo (féminisme, écologisme, communalisme, etc.). Par le brouillage des signaux, cette valorisation symbolique d'une opération tactique géostratégique assez controversée, visant essentiellement à contrer toute emprise turque sur la région, impliquait la mise en avant d'un discours révolutionnaire progressiste faisant la part belle au concept de liberté $\underline{60}$. Parallèlement à cela, l'usage du terme azadî a connu un itinéraire long et complexe dans les productions discursives et artistiques au sein de la mouvance du PKK, active essentiellement en Turquie et au nord de l'Irak. Ce fond a nourri de manière importante les discours en vigueur au Rojava et a permis au PYD/PKK de quadriller le territoire avec une nouvelle grammaire politique et esthétique. Entre autres exemples, la place publique située au centre de la ville de Kobane et l'unité administrative (Kanton) qui couvre la région de Derîk ont, toutes deux, été rebaptisées azadî. Des fresques affublées de ces cinq mêmes lettres font leur apparition sur les murs des villes du Rojava. L'arche qui se trouve à l'entrée de la ville de Tirbe Spî porte depuis quelques années la mention « Her tişt ji bo Azadiye » (« Tout pour la liberté ») $\underline{61}$ qui n'est pas sans rappeler le titre d'un film du collectif kurde « Komîna fillma Rojava », un groupe de cinéastes et de techniciens du cinéma kurdes de Syrie réfugiés dès 2012 en Turquie puis en Allemagne, qui célèbre la résistance kurde lors de l'offensive de l'armée turque en 2015 contre Sûr, un quartier de la ville de Diyarbakir (Amed) en Turquie $\underline{62}$.

\section{Visit the web version of this article to view interactive content.}

Entrée de la ville de Tirbe Spî décorée du slogan «Tout pour la liberté » (Her tişt j ibo azadiye).

https://web.archive.org/web/20210718200444/https://twitter.com/Nuvinlbr ahim/status/1416851428157579264 
Tous ces signaux contribuent à produire une forte indistinction à la fois entre action politique et action militaire et entre les différents terrains sur lesquels la mouvance du PKK exerce celles-ci. Cette fusion s'exprime le plus nettement dans la mise en avant du rôle romanticisé des combattantes kurdes des YPJ (Yekîniyên Parastina Jin, Unités de protection des femmes) dont le cri de guerre « Jin, jiyan, Azadî » (« la femme, la vie, la liberté ») apparu vers 2013 est porté aux nues au sein des gauches internationales et dans la sphère médiatique occidentale en lien avec la résistance de Kobane contre Daesh et la révolution du Rojava $\underline{63}$. Dès lors le terme azadî échappe à toute logique locale pour devenir l'emblème positif d'un combat contre l'obscurantisme et le patriarcat. Recenser et analyser les productions scientifiques, journalistiques, littéraires, artistiques et cinématographiques occidentales faisant référence au Rojava de 2012 à nos jours représente un travail considérable. Nous ne citerons ici que quelques exemples marquants soulignant la reproduction du terme azadî dans une multitude de sphères à l'international. Tout d'abord, on peut noter la continuité et le renouvellement de la projection des luttes kurdes au sein des gauches européennes. À l'instar de l'expérience du Chiapas, le combat du Rojava a eu un rôle de catalyseur dans certains milieux progressistes occidentaux donnant un sens concret à des engagements politiques relativement troublés par l'affaiblissement du socialisme et des mouvements dits altermondialistes. L'imaginaire et le décorum kurdes prennent une place importante dans ces espaces militants. Du festival de Mauléon, au Pays basque français, haut lieu de la culture militante gauchiste, à Bologne en passant par Berlin, Londres ou Vienne, on ne compte plus les évènements et les manifestations en faveur du Rojava, qui sont autant d'occasions de scander et reproduire sur les affiches et les fresques géantes le fameux slogan « Jin Jiyan Azadî » (la femme, la vie, la liberté) $\underline{64}$. Il existe également une multitude d'ouvrages ou de communications orales situés entre publications scientifiques et œuvres militantes faisant la part belle au terme azadî. Prenons par exemple, l'intervention à propos de l'avenir du Rojava par le célèbre intellectuel américain, Noam Chomsky, intitulée «Azadiya Rojava », en 2021 65 . 


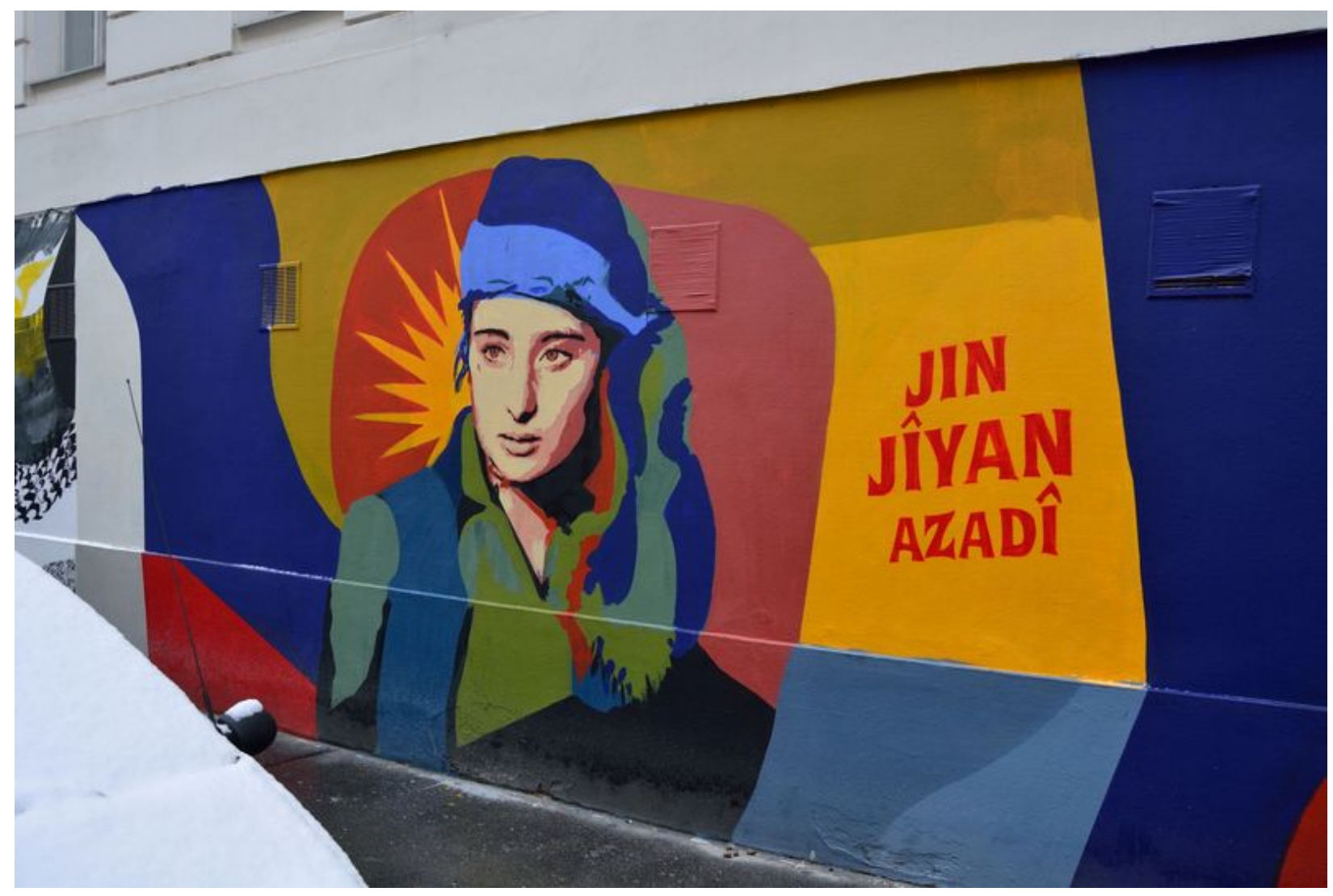

Le plus célèbre slogan du mouvement kurde « Jin Jiyan Azadî » (La femme, la vie, la liberté) sur un mur de Vienne, en janvier 2021. Crédit Maryam Ashrafi. Source : https://commons.wikimedia.org/wiki/File:Jin_Jiyan_Azadi_by_Btoy,_Schwendergasse , Vienna.jpg 


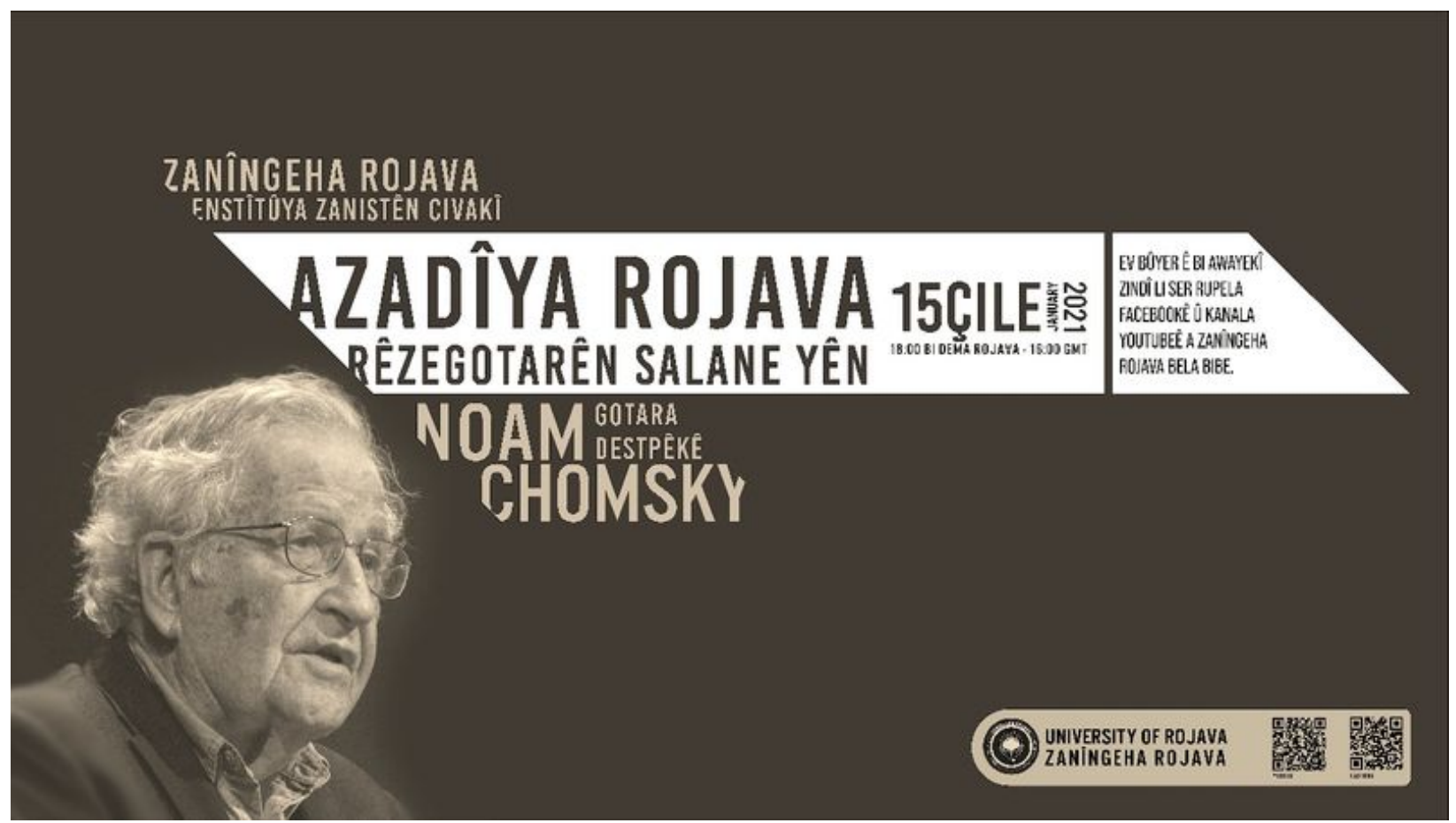

Affiche invitant à la conférence du professeur Noam Chomsky à I'Université du Rojava en janvier 2021.

L'espace indistinct des productions audiovisuelles contribue également à relayer les images de l'évidence de la révolution du Rojava. Reportages journalistiques et fictions militantes teintés de romantisme convergent soit pour défendre de manière explicite le projet politique en vigueur au Rojava soit pour présenter une toile de fond monologique de la situation sur place. Le continuum est saisissant lorsque tour à tour des groupes de femmes (combattantes ou comédiennes) scandent le slogan « Jin, Jiyan, Azadî » pour la caméra de la journaliste Mylène Sauloy en 2016 $\underline{66}$, sur le tournage des filles du soleil de la cinéaste Eva Husson, ou au sortir de la projection de ce même film lors du Festival de Cannes en 2018드. Dans la même veine et la même année, le magazine Vogue Italia publie une série de photos de combattantes du Sinjar et du Rojava dont « le mouvement féministe kurde » serait, nous explique-t-on, « le mouvement de soutien aux droits des femmes le plus puissant du monde ». Outre les grosses productions cinématographiques, on trouve un grand nombre de séries dans lesquelles le Rojava et les Kurdes sont évoqués sans entrer dans les détails et où le mot azadî figure en bonne place. Dans No man's land, une mini-série diffusée en 2020 sur Arte, le frère d'une archéologue française présumée disparue croit reconnaître sa sœur dans un reportage de France 24 sur des combattants internationaux ayant rejoints les YPG. La personne interviewée dans le reportage fictif crie le slogan « Jin Jiyan Azadî » avant de périr sous le feu d’un tir de mortier $\underline{68}$. Il n’y a pas ici de propos 
politique ou militant, mais bien la démonstration du fait que certaines formulations militantes kurdes font dorénavant partie d'un paysage audiovisuel et d'une culture mainstream internationale. La même année, la série Stateless met en scène une ressortissante australienne qui, se faisant passer pour une réfugiée, est internée avec des exilés afghans, kurdes et srilankais dans un centre de rétention illégal. Dans le quatrième épisode de la première saison, une militante associative organise une manifestation pour la libération des dits réfugiés au cri de «azadî, freedom ! » $\underline{69}$. Azadî est un terme commun à la langue kurde, au persan dari et au pashto parlés en Afghanistan. Il devient ainsi un mot international de la langue de la rébellion. La mise en abîme, ou plutôt la fusion de toutes les dimensions du réel est remarquable. Les spectateurs occidentaux se regardent eux-mêmes avoir de la compassion et protester pour les réfugiés kurdes et afghans et parler dans leur langue.

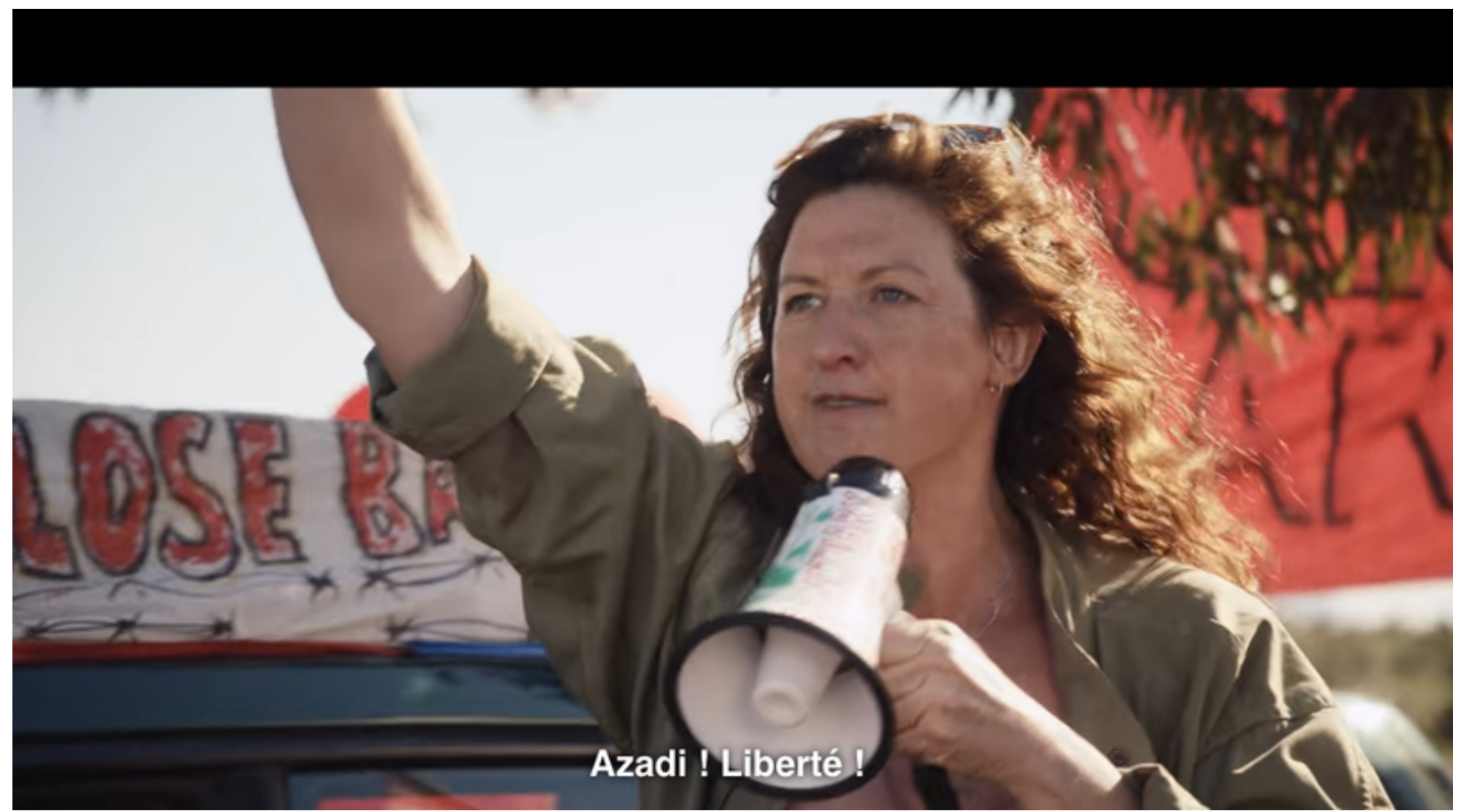

Dans Stateless, une série télévisée australienne, Kate Box joue le rôle d'une activiste engagée auprès des réfugiés afghans et kurdes. (Capture d'écran)

Azadî est également devenu un label commercial dans le monde des jeux de rôle en ligne : sur une de ses plateformes, on peut télécharger les modèles à imprimer en 3D des figurines du Azadî Death Front, une armée de féroces combattantes $\underline{70}$. Rien n'indique que ces « féroces combattantes » soient kurdes, en dépit d'une esthétique et d'un titre clairement identifiable. L'opération commerciale - peut-être juteuse - se faisait sous couvert de la défense de la cause des femmes en général: un appel à dons 
à l'attention de la communauté des joueurs, mettait en avant la nécessité de féminiser la gamme des guerriers représentés dans ce type de jeux.

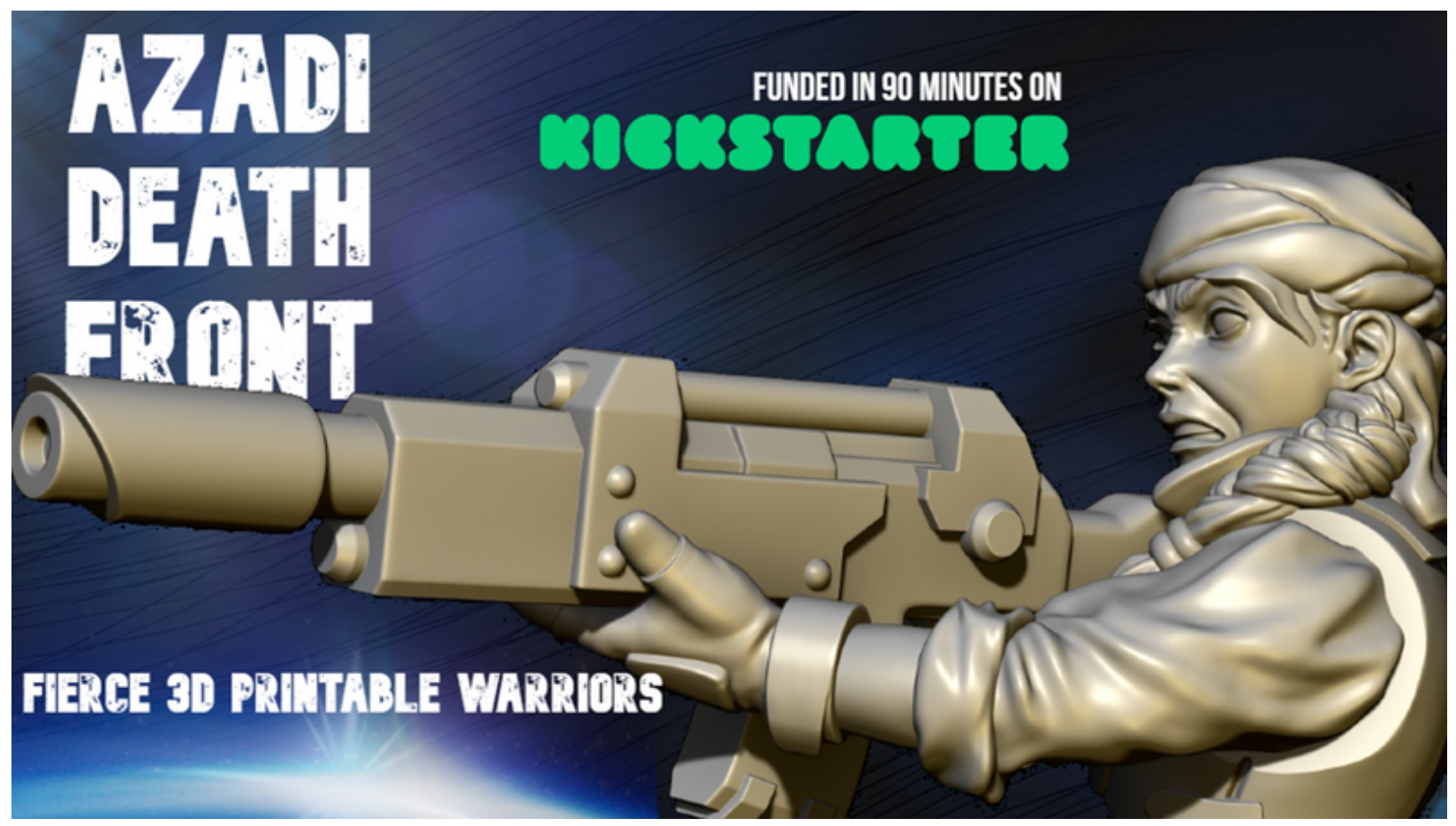

Publicité pour les modèles de figurine de jeu de rôle Azadî Death Front

Photoreportages, bandes dessinées $\underline{71}$... musique électronique $\underline{72}$, nous abreuvent d'images et de thèmes rassurants ou galvanisants. Dans tous les cas, les lourdeurs et la complexité de la crise syrienne, du jeu géopolitique, de l’histoire et de la structuration de l'organisation dont sont issues les fameuses combattantes, à savoir le PKK, voire la question féministe s'effacent pour laisser place à un spectacle cathartique univoque, mais tout à fait étranger à la situation locale. Cette réappropriation culturelle au mépris des réalités du terrain et d'une situation féminine complexe n'est pas sans susciter débats et critiques dans les rangs des plus fervents défenseurs des YPJ그.

Azadî est aujourd'hui le terme kurde le plus connu en Occident, mais il est difficile de savoir ce qu'il veut dire pour ceux qui le profèrent et ceux qui l'entendent. S'agit-il d'un slogan nationaliste kurde, d'un cri de guerre féministe, ou d'une protestation en faveur des réfugiés ? En s'internationalisant, il semble avoir perdu la densité acquise sur la longue durée dans les milieux kurdes, au sein du PKK et dans d'autres formations politiques et lors d'une brève irruption au sein de l'espace contestataire kurdo-arabe de la révolte syrienne de 2011. Sa diffusion est en tout cas le symbole du 
paradoxe de la visibilité de la présence kurde qui dépasse largement les frontières du Moyen-Orient alors qu'elle peine encore à être acceptée en Syrie.

\section{Bibliographie}

La bibliographie de cet article est disponible dans le groupe Zotero du Lexique (https://www.zotero.org/groups/4550572/syria lexicon/collections/ZRSKEYV4).

\section{Footnotes}

1. Nous pensons notamment à la grammaire kurde du missionnaire italien Maurizio Garzoni du XVIII ${ }^{\mathrm{e}}$ siècle qui mentionne l'adjectif « libre » (aza) et le verbe « se libérer » (xelas kirin) et au vocabulaire anglais-arabe-persan-turc-arménien-kurdesyriaque compilé par les services de renseignement de la Royal Navy en 1920, qui comprend aussi uniquement l'adjectif « libre » (azad). Garzoni, Maurizio. Grammatica e vocabolario della lingua kurda ... Roma : Sacra Congregazione di Propaganda Fide, 1787. http://archive.org/details/b28777086. ; Great Britain. Admiralty. Vocabularies: English, Arabic, Persian, Turkish, Armenian, Kurdish, Syriac. London, H.M. Stationery Off., Frederick Hall [printer] Oxford, 1920. http://archive.org/details/vocabulariesengl00grearich.

2. Yüksel, Metin. 2021. "Kurds and Kurdish Nationalism in the Interwar Period.” In The Cambridge History of the Kurds, 205-28. Cambridge University Press. https://doi.org/10.1017/9781108623711.008.

3. Tejel, Jordi. Syria's Kurds. Routledge, 2008. https://doi.org/10.4324/9780203892114. p. 26. 4.

Cavaillès, Sylvain. «La littérature comme instrument de sauvegarde du patrimoine culturel immatériel des Kurdes de Turquie ». Anatoli. De l'Adriatique à la Caspienne. Territoires, Politique, Sociétés, no 6 (1 octobre 2015): 157-73. https://doi.org/10.4000/anatoli.299.

Rehmany, Wirya. Dictionnaire politique et historique des Kurdes. Paris: L'Harmattan, 2014, 162. http://www.worldcat.org/oclc/891786435.

5. Parmi les poèmes d'amour, les odes mystiques et épiques et les poésies contant la vie villageoise kurde, le premier recueil (Agir û pirûsk ou Prûsk û petî) fait surtout l'éloge de la patrie perdue («Bêriya welat » / « Ka Welat û Al û Ol » ; « Kurdistanim ka ? ») et des figures kurdes révoltées (« Tarîxa Şex Seidê Rehmetî »). Il évoque la 
souffrance face à l'oppression (turque) et à la division et appelle à l'unité et à la guerre. Ces éléments à teneur mélancolique et politique fusionnent dans le personnage romantique que se construit Şexmûs Hesen (« Ji Kulan Bûme Cegerxwîn » Des souffrances, je suis devenu Cegerxwîn ; Seulement Cegerxwîn « Cegerxwîn Bes »; « Dilê Cegerxwîn » etc.) - Cegerxwîn veut dire « Cour meurtri ». Seule une petite élégie fait référence à la liberté de manière allusive et ironique « Nous ne sommes pas des esclaves » (Em ne kole ne !). Il y clame le refus d'être assujetti à l'objet de son combat politique : Ô mon pays ! Nous sommes tout affairés à répondre à tes ordres / Nous sommes tes jeunes ouvriers journaliers ; mais nous ne sommes ni tes serfs, ni tes esclaves. Cegerxwîn. Agir û Pirûsk: Dîwan 1, 2014, 119. http://www.worldcat.org/oclc/1121100744.

6. Il s'agit là de noms issus de la titulature ottomane désignant à l'origine des chefs militaires et qui prirent le sens d'autorités quasi-féodales. $\_$

7. Cigerxwîn. Sewra Azadî : Dîwan 2, 2014, 113-116.

http://www.worldcat.org/oclc/1110445879.

8. Tejel, Jordi. «The Kurdish Question in Syria, 1946-2019 ». In The Cambridge History of the Kurds, édité par Hamit Bozarslan, Cengiz Gunes, et Veli Yadirgi, $1^{\text {re }}$ éd., 436-57. Cambridge University Press, 2021. https://doi.org/10.1017/9781108623711.018. 9. JAMES, Boris. « Déconstruire le moment kurde : entre pankurdisme, fragmentation et adversité géopolitique », 4 septembre 2020. https://www.africt.org/article/deconstruire-le-moment-kurde-entre-pankurdisme-fragmentation-etadversite-geopolitique/. $\leftrightarrows$

10. Cf. Bibliothèque numérique kurde. «Azadî, $n^{\circ} 1$ ». Bibliothèque numérique. Consulté le 4 janvier 2022. https://bnk.institutkurde.org/catalogue/detail.php? pirtuk=535. ; Portail mondial des revues. « Azadi ». Base de données. Consulté le 4 janvier 2022. https://sismo.inha.fr/s/fr/item/6428.

11. White, Paul J. The PKK: Coming down from the Mountains, 2015. http://www.worldcat.org/oclc/1003411901. ; Grojean, Olivier. La Révolution kurde: Le PKK et la fabrique d'une utopie. Cahiers libres. La Découverte, 2017. https://doi.org/10.3917/dec.groje.2017.01. 12. Apo va jusqu'à nier publiquement l'existence d'un peuplement kurde originaire en Syrie et le problème politique qui en découle. Jordi Tejel, Syria’s Kurds, op. cit. p. 78. 


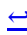

13. Cf. Photo, “New Roz à Kobane” ('Ayn al-'Arab), mars 2007, collection personnelle. $\underline{-}$

14. Tejel Gorgas, Jordi. « Les Kurdes de Syrie, de la "dissimulation” à la "visibilité" ? » Revue des mondes musulmans et de la Méditerranée, no 115-116 (31 décembre 2006). https://doi.org/10.4000/remmm.3022. 15. Jordi Tejel, Syria's Kurds, op. cit. p.110-114. 16. Jordi Tejel, Syria's Kurds, op. cit. p. 114-133;Gauthier, Julie. «Syrie : le facteur kurde ». Outre-Terre 14, no 1 (2006): 217. https://doi.org/10.3917/oute.014.0217. ; Gauthier, Julie. «Les événements de Qamichlo : irruption de la question kurde en Syrie? »Études Kurdes, n ${ }^{0} 7$ (mai 2005).

https://web.archive.org/web/20210127105915/https://www.institutkurde.org/publicati ons/etude kurdes/etude7.php.p. برس 23 Press. Belgeyên Serhildan Qamişlo أنتفاضة القامشلي 2004 وثائقي حصريا من برس 23 برس 2016 2016:: 2004/3/12. يلفزيون سوريا. انتفاضة . 2019 . 20 17. حسان حسن. أحداث القامشلي 1232004 توجه المنتفضين الى وسط مدينة قامشلو, 2012 . https://www.youtube.com/watch?v=6C1YdSBGkvA. ; Omar Ali. مليونية تشيبع شهداء مايونة https://www.youtube.com/watch?v=IPmZGcjE1M. ; lilhill2002. Syria protest - in Qamishli, 12.03 « Serhildan » Demo, 2011. https://www.youtube.com/watch?v=OjnWKr7XneI.. 18. (à 10'34") jindires. 2004-3-17 عفرين Efrîn-Raperîn, 2016. https://www.youtube.com/watch?v=dAkUzEBnK9c.. 19. ShaamNetwork S.N.N. شام - الشيخ معشوق الخزنوي بتأبين شهداء الكرد 2004, 2011. https://www.youtube.com/watch?v=xzE1gqso7ZA. 20. Jordi Tejel, Syria's Kurds, op. cit. p. 115-116. 21. Jordi Tejel, Syria's Kurds, op. cit. p.128. 22. Schmidinger, Thomas. Rojava: Revolution, War and the Future of Syria's Kurds. London: Pluto Press, 2018. http://www.worldcat.org/oclc/1082376427. p. 85. 
23. cane11kurd. مظاهرة قامشلو في جمعة ازادي 29.04.2011 جمعة الغضب, 2011. https://www.youtube.com/watch?v=XFcNqfusS8g. $\_$

24. Trois pages facebook attirent notre attention, celle du Tevgara ciwanên kurd (le mouvement des jeunes Kurdes), celle de Akrem Uso, un photographe indépendant et activiste kurde originaire de Derbasiyyeh, et celle de la Şoreşa ciwanêne kurd li surya (la révolution des jeunes Kurdes en Syrie). Facebook. « Tevgra ciwanên kurd ( Tركة الشبـــــاب الكورد ( . C . K ) ». Réseau social. Consulté le 4 janvier 2022. https://www.facebook.com/Ciwanekurd.net1/photos/?ref=page internal. ; Facebook. 《Şoreşa Ciwanên kurd li sûrya - ثورة شباب الكورد السوريين 》. Réseau social. Consulté le 4 janvier 2022.

https://www.facebook.com/groups/shabab.alderbasya/about/. ; Facebook. " Group akrem uso ». Réseau social. Consulté le 4 janvier 2022.

https://www.facebook.com/LensKurdishYoungL. Elles sont loin de rassembler toutes les initiatives kurdes et la présence des activistes kurdes se fait également ressentir sur les pages Facebook communes de veille de la révolution syrienne. https://www.facebook.com/groups/253771082017313/ 25. Cf. Facebook. "Group akrem uso - جمعة أمريكا..... ألم يشع حقدكم من دمائنا 19/10/2012 ». Réseau social. Consulté le 4 janvier 2022. https://www.facebook.com/LensKurdishYoung/photos/a.104377973042553/130333677 113649/. ; Facebook. " Group akrem uso - -10-2012 مظاهرة الدرباسية اول ايام عيد الاضحى 26 | Facebook ». Réseau social. Consulté le 4 janvier 2022. https://www.facebook.com/LensKurdishYoung/photos/a.104377973042553/132306046 916412/. ; Facebook. « Group akrem uso - جديد: كتاب الحرية freedom في مظاهرة الدرباسية 22-10-2012 ». Réseau social. Consulté le 4 janvier 2022.

https://www.facebook.com/LensKurdishYoung/photos/a.104377973042553/131251453 688538/.

26. Entretien de l'auteur avec Sardar Mullah Darwish, 01/09/2021.

27. Facebook. «Group akrem uso - 20-5-2011 الصورة بعدسة شاب كوردي في جمعة ازادي عدسة شاب كوردي تنقل لكم الوقائع http://www.facebook.com/LensKurdishYoung الدرباسية الدورية وعبر سكايب helar555@hotmail.com من ارض الحدث بكل مصداقية وجرأة --لتواصل معنا zorava5 ». Réseau social. Consulté le 4 janvier 2022. https://www.facebook.com/LensKurdishYoung/photos/a.104377973042553/195016533

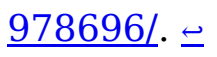


28. ShaamNetwork S.N.N. شام - القامشلي في جمعة الشهداء 1-41-4011, 2011. https://www.youtube.com/watch?v=mThzlLM-ruc. ; rasto rastu. عامودا.جمعة معتقلي في 2012-1-20 الثورة.flv, 2012. https://www.youtube.com/watch?v=rIOFtM3OkGk.. 29. مؤسسة الشارع للإعلام. A tribute to Meshal Temo تحية الى مشعل تمو, 2012. https://www.youtube.com/watch?v=RvvKBekT2Fk. ; dengizcan@gmail.com. فيلم 年. https://www.youtube.com/watch? v=ONCMMcmkvZw. ; Assad Ordered Killing of Kurdish Activist Mashaal Tammo Leaked Files, 2012. https://english.alarabiya.net/News/2012/10/10/Assad-orderedkilling-of-Kurdish-activist-Mashaal-Tammo-Leaked-files. ; خاز, أزادي. 》 إلى روح الشهيد 》. Creative Memory. Consulté le 4 janvier 2022.

https://creativememory.org/ar/archives/? fwp_searchwp_archives=\%D8\%A3\%D8\%B2\%D8\%A7\%D8\%AF\%D9\%8A\&fwp_categor $\underline{\text { ies }=\text { music-singing-ar. }} . \underline{\underline{ }}$

30. jihadmmm. الحسكة الدرباسية آزادي آزادي مظاهرات جمعة الحظر 28 10, 2011 . https://www.youtube.com/watch?v=57KaXtbollM. ; Facebook. « Group akrem uso -

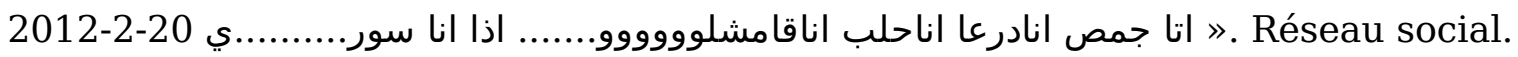
Consulté le 4 janvier 2022. https://www.facebook.com/LensKurdishYoung/photos/a.104377973042553/106985259 448491/.

31. Entretien de l'auteur avec Sardar Mullah Darwish, 01/09/2021 et Seve AydinIzouli, 05/11/2020.

32. https://www.youtube.com/watch?

$\mathrm{v}=$ sHivMmmCBhU\&list $=$ RDsHivMmmCBhU\&start_radio=1

33. Sivanistan. "El Selam wel Hurriye» (Azadî û Aşitî) Sivan Perwer 2011, 2011. https://www.youtube.com/watch?v=-nq0p6HIOLE.

34. Rahman Mumtaz. سميح شقير - لي صديق من كردستان, 2012. https://www.youtube.com/watch?v=Xwb_2gB8s5M.

35. Entretien avec l'auteur, 04/12/2020. À écouter également, le récit par l'essayiste kurde de Syrie, Loqman Derkî, d'une soirée de concerts de Samih Shqayr à Alep : SouriaLi Radio. ألف سردة وسردة 170 - لي صديق من كردستان, 2015. https://www.youtube.com/watch?v=dErSdzUmyNE. 
36. freesyriaobas. سميح شقير في المانيا بون|07|09. 2011, 2011.

https://www.youtube.com/watch?v=CkMQfXwges4..

37.

العلواني, عبد المجيد. » الجمعة العاشرة: شيخ قراء الشام يستقيل والأسد يخفض سعر الماوزت «. أورينت mars 2013. https://web.archive.org/web/20220105105220/https://orientnews.net/ar/news_show/2681/0/.

Facebook. 《جمعة ازادي 》. Réseau social, 20 mai 2011.

https://www.facebook.com/photo/?

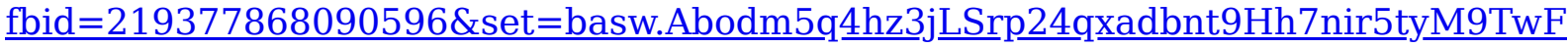
W-lOEsEAlxJuJ 4vtegJ xXbzNJEqklFSi-AdvM -RVy8ZE2NsTnishI60Nf6jW9sNTRuKogvje1ZL3B5OL_MkT4AQUeHb31vpV6aF8FLMStk373chb_OPY_QM8g_Hd7gSr2Ck6EGDUdycHGbpbMaBOYQCRyj83-6Cq06MmUt9PLw-

W6\&opaqueCursor=AbodG7DlwAUaIEpcO3C-

dhy8i26mUz0n3q_ua0LG4FPM8zoWBqYbig-xrOMPef9l-

RvwpYekoiwbuvFUXSUeVit1fLw7m0pUoB2GroEQuz9IgJNdVLDipvqU00iQDWWCb7Z igMzAe-qedEeD9wOrLSphugNLHJIvTwh2zgt-

MT2EiBrlL1dMlloJIh5l5e5jLECydd9F7IJR4q-yT3UspTuwE5Zbutr2sCoL3Zd-

45bKwZwbLOnhtkD3aBneF0Yk1u cEVDJxFGA-

WCp8TQTubBfRyh47CXGhgy5WVMzJzHK239XL617qmIxrJ1xenIlx8E15ktxEMwKTKM 9NUIIvtGF84HMSgm uJggVmF7cpRsEbUCwvMLPCOzVyYCnBL5zzino24nRTsSrRRY 9KLBo3wIy3hKjOGXKavfF75-

st4Oz1x0nzcTOCO20 7AOHPBsgYEKhsn3MQ5QE Lci7jTyPr7ZoXwlB8fRNhXfJagg 351 GbZXP1qPXqxAOJeUvnx6yGylmBb9 PcAdJvYl7dJJvePUdZ8IzRREMtZQnjjtxF6FdJOyJ. VnjtxnkMo7n7IIBtlDs2VcOvDBkxNumx6uI0O3F65865NbZ QWdnUpfuogUdbU YLP4 Ow8gphejy27 2PQDWc3n6YZzjSBPnwGrvPO3moBeot7FAE4sCbd 72gc45fFy 6 J bLkb Mcmx1izRDmGfHsxL4coc5RxfhkaDfZ9QfX91pE4I T2Le 6gCqK1CYOvfu1tAtdRlizoE vEgq2CqEJENzv5ZOXmUhFlYDNYc. $\triangleq$

38. Facebook. "Group akrem uso - لن ننسى جمعة ازادي الذي يصادف اليوم 2011-50 شباب الكرد تحيي من اختار جمعة ازادي بعدسة شاب كوردي

http://www.facebook.com/LensKurdishYoung عدسة شاب كوردي تنقل لكم الوقائع من ارض zorava5 ». Réseau social, 20 mai 2013.

https://www.facebook.com/LensKurdishYoung/photos/a.104377973042553/200269406

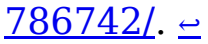


39. Ecouter Sana Yazigi qui lors de l'émission de la Grande table des idées de France Culture indique qu'en Syrie on dit aussi « azadî » pour dire liberté, « parce qu'on est kurde et arabe en Syrie ». À 8'50", « Printemps arabes : la révolution des mots ? » France Culture. Consulté le 31 décembre 2021.

https://www.franceculture.fr/emissions/la-grande-table-idees/printemps-arabes-larevolution-des-mots. $\bullet$

40.

(Images du vendredi Salih al-'Ali à Berzeh), ShaamNetwork S.N.N. شام دمشق برزة  $\underline{\mathrm{v}=1 \mathrm{HQ}} \underline{\mathrm{kQ}} \underline{0 \mathrm{aK}} \underline{\underline{\mathrm{bs}}}$.

(Evocation du bataillon Sultan al-Atrash) Mémoire créative de la révolution syrienne. Chroniques de la révolte syrienne: Des lieux et des hommes 2011-2015. Presses de l'Ifpo, 2018. https://doi.org/10.4000/books.ifpo.12765. p. 191

Rey, Matthieu, et Laura Ruiz de Elvira. « Luttes de sens, cadrages et grammaire lexicale en contexte révolutionnaire. Le cas de la Syrie (2011-2012) ». Cultures \& conflits, $\mathrm{n}^{0} 117$ (1 juillet 2020): 11-33. https://doi.org/10.4000/conflits.21372. p. 17. 41. Dakhli, Leyla. L'esprit de la révolte : archives et actualité des révolutions arabes, 2020. http://www.worldcat.org/oclc/1220977041.

42. Allfor Syria. قامشلو جمعة أزادي 2011-05-2011, 201//www.youtube.com/watch? $\underline{\mathrm{V}=\mathrm{J}} \underline{4 \mathrm{P} \times 8 \mathrm{eHBc} 4}$.

43. ShaamNetwork S.N.N. شام - عامودا - مظاهرات جمعة آزادي 5-20 ج1, 2011. https://www.youtube.com/watch?v=dPhR9RT5d_w. 44.

ShaamNetwork S.N.N. 1 شام الحسكه عامودا اغنية الف وبي بحنجرة بلبلة الثورة في عامودا 25 2012 ,2012. https://www.youtube.com/watch?v=6UblG_pNSjw.

Lila TV. Aleph Be Boubeye $\square 2018$ ألف باء بوباية (19.19). https://www.youtube.com/watch?v=4w4GMjuIYTU.. 45. Thomas Schmidinger, Rojava, op. cit., p. 86-110. 46. En 2012, on voit les combattants de la Brigade Meshaal Temo, brandissant les drapeaux de la révolution syrienne et du Kurdistan, scander en arabe à la fois «Allahu Akbar » et « Azadî pour l'éternité que tu le veuilles ou non, Assad ! ». sham 
qamshlo Sham. مشاركة لواء مشعل مع عناصر الجيش الحر ورفع العلم الكردي, 2012.

https://www.youtube.com/watch?v=6VIRmJt4N_g. $\doteq$

47. MENA. الفصائل العسكرية الكردية في الثورة السورية.. التوجه والانتشار والموقف من التطورات

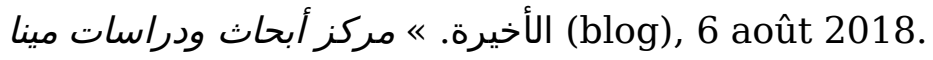

https://web.archive.org/web/20220105131012/https://mena-

studies.org/ar/\%D8\%A7\%D9\%84\%D9\%81\%D8\%B5\%D8\%A7\%D8\%A6\%D9\%84-

\%D8\%A7\%D9\%84\%D8\%B9\%D8\%B3\%D9\%83\%D8\%B1\%D9\%8A\%D8\%A9-

\%D8\%A7\%D9\%84\%D9\%83\%D8\%B1\%D8\%AF\%D9\%8A\%D8\%A9-\%D9\%81\%D9\%8A-

\%D8\%A7\%D9\%84\%D8\%AB\%D9\%88\%D8\%B1\%D8\%A9-\%D8\%A7\%D9\%84/. .

48.

قناة حلب اليوم. لقاء خاص مع أزاد شعبو قائد كتيبة ازادي الكردية في جيش Halab Today TV

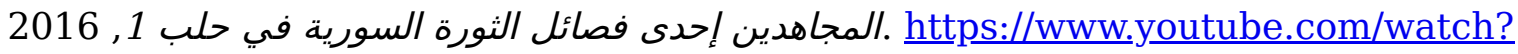
$\mathrm{v}=\mathrm{UGeD} 8 \mathrm{E} y \underline{2 \mathrm{r} 9 \mathrm{~s}}$.

saif al deen shaabo. كتيبة آزادي وبيان الأنضمام للمجلس العسكري الكردي, 2015. https://www.youtube.com/watch?v=pGtmKghQ $\underline{5 n c}$.

49.

Quelques autres groupes armés kurdes comportent dans leur appellation le terme « azadî ». Tous revendiquent leur participation dans la révolution syrienne et tous expriment leur hostilité vis-à-vis du régime syrien et de ses collaborateurs (shabbiha) souvent désignés comme étant les membres du PYD/PKK.

Yusef Azmeh. كتيبة صقور آزادي, 2013 https://www.youtube.com/watch?v=EoNVr9-M0Kk qamshloo32. تشكيل كتائب ازادي, 2012. https://www.youtube.com/watch?

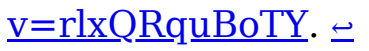

50. Entretiens avec l'auteur, 2020, 2021. 51. 
Entre autres exemples : à Hajar al-Aswad et à Qatana, à la périphérie de Damas, les دمشق .الحجر الأسود . دمanifestants évitent de prononcer le terme 《azadî » : golaan1967 2011-5-20 جمعة الحرية ج1..flv, 2011. https://www.youtube.com/watch?v=2uEsvaRohk0.

zlzalzlzal. الثورة السورية قطنا ريف دمشق 2052011 جمعة أزادي الحرية ج1, 2011 جال. https://www.youtube.com/watch?v=UuHEguAV2B4.

zlzalzlzal. الثورة السورية قطنا ريف دمشق 2052011 جمعة أزادي الحرية ج2, 2011 ج. https://www.youtube.com/watch?v=YW9qcAfFASo.

52. 14march.org. مقتل 27 شخص في جمعة ازادي - الحرية, 2011. https://www.Youtube.com/watch?v=kcFA6KqjXRY.. 53.

Libération. « Au moins 44 morts en Syrie ». Libération, 20 mai 2011, sect. International.

https://web.archive.org/web/20220105132020/https://www.liberation.fr/planete/2011/ 05/20/au-moins-44-morts-en-syrie 737166/.

Mémoire créative de la révolution syrienne. Chroniques de la révolte syrienne: Des lieux et des hommes 2011-2015. Presses de l'Ifpo, 2018.

https://doi.org/10.4000/books.ifpo.12765. p. 109.

54. En janvier 2012, lors du Vendredi des prisonniers politiques, une banderole indique "le fédéralisme est notre revendication » : (à 0'15”) Partya pêşverû. مظاهرة شامود/"جمعة معتقلي الثورة" , 2012 . https://www.youtube.com/watch?v=oVWKqIpE-E4.

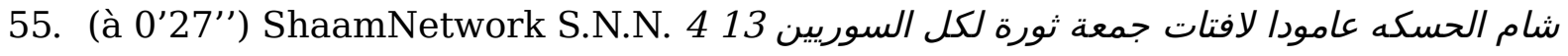
2012 ,2012. https://www.youtube.com/watch?v=si1XQs21ouM.

56. Welatê Me. «حوار مع رئيس المجلس الوطني السوري د. برهان غليون 》. Webzine. Consulté le 5 janvier 2022.

https://web.archive.org/web/20220105132805/http://welateme.net/erebi/modules.php ?name $=$ News\&file $=$ article\&sid $=12437 \#$. YdWc-lm2zb2.. 57. Sherif omeri. sherif omeri Azadi شريف اومري ازادي, 2017. https://www.youtube.com/watch?v=ube_rYhGvdQ.. 58. ZINDî - Li Qamişlo Raperîna 12ê Adarê tê bibîranîn. Rudaw Kurmanci, 2020. https://www.facebook.com/kurmancirudaw/videos/615317512655497/. 
59. Entretien avec l'auteur, 15/11/2021.

60. Suleiman Rached. SOZA DOZÊ- ŞOREŞA ROJAVA- ŞERVANÊN AZADIYÊ, 2015. https://www.youtube.com/watch?v=4F7EDgG9ZsY.

61. Ibrahim, Nuvin. « \#Status4NorthAndEastSyria Her tişt ji bo Azadiyê For our freedom, support Rojava ». Réseau social. Twitter, 18 juillet 2021.

https://web.archive.org/web/20210718200444/https://twitter.com/NuvinIbrahim/statu s/1416851428157579264.

62.

Facebook. « Komîna fîlm a Rojava ». Réseau social. Consulté le 5 janvier 2022. https://www.facebook.com/rojavafilmcommune/.

KEDISTAN. « Ji bo Azadiyê • Un film sur les silences hypocrites de 2015 ». Webzine, 24 mai 2020.

https://web.archive.org/web/20210116090252/https://www.kedistan.net/2020/05/24/ji -bo-azadiye-film-sur-silences-hypocrites/.

Irigoyen, Lucile. « Le cinéma au Rojava, un outil politique, culturel et social ». Cahier d'Histoire immédiate, $\mathrm{n}^{0} 56$ (novembre 2021).

http://www.worldcat.org/oclc/44071976.

63. James, Boris. « Les années PKK ». En attendant Nadeau (blog), 22 février 2018. https://web.archive.org/web/20210423021733/https://www.en-attendantnadeau.fr/2018/02/22/annees-pkk/.

64. Aktaş, Ercan. « From Rojava to the Basque country: The road of solidarity ». KEDISTAN, 23 octobre 2021.

https://web.archive.org/web/20211023173810/https://www.kedistan.net/2021/10/23/fr om-rojava-to-basque-country $l . \pm$

65.

Kurd Events. « Rojava Freedom Annual Lecture Series Inaugural Lecture: Noam Chomsky ». Consulté le 5 janvier 2022.

https://web.archive.org/web/20220105140822/https://kurdevents.com/event/rojavafreedom-annual-lecture-series-inaugural-lecture-noam-chomsky 1 .

Rojava University. Rojava Freedom Annual Lecture Series - Inaugural Lecture: Noam Chomsky, 2021. https://www.youtube.com/watch?v=R2WSbzCevAs.. 
66. (à 4'07) Cognacaise PCF16. Kurdistan, la guerre des filles - Arte 8 mars 2016, 2016. https://www.youtube.com/watch?v=WWdnAW83KTA. Sauloy, Mylène.

Kurdistan, la guerre des filles, 2016.

https://web.archive.org/web/20201204180951/https://www.magnetotv.com/fr/docume ntary/kurdistan/.. 67.

(à 1'43) wildsidecats. LES FILLES DU SOLEIL - Bande-annonce (VF), 2019. https://www.youtube.com/watch?v=sr8Sz01VY1M.

(à 4'09) CANAL+ Cinéma. Sortie du film Les Filles du Soleil d'Eva Husson - Cannes 2018, 2018. https://www.youtube.com/watch?v=4Q7rANq5sU8. 68. « No Man's Land (mini-série) ». In Wikipédia, 23 décembre 2021. https://fr.wikipedia.org/w/index.php?title=No_Man\%27s_Land_(mini-

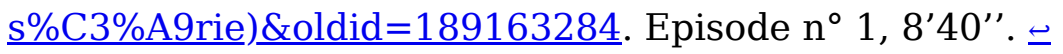

69. «Stateless (série télévisée) ». In Wikipédia, 9 novembre 2021.

https://fr.wikipedia.org/w/index.php? title=Stateless_(s\%C3\%A9rie t\%C3\%A91\%C3\%A9vis\%C3\%A9e)\&oldid $=187837376$. Episode $n^{\circ} 4$ saison 1, 6’16”. 70.

Debutify. « Azadi Death Front Premium Army Deal - STL for 3D Printing ». 40Emperor. Consulté le 5 janvier 2022. https://web.archive.org/web/20210126124745/https://40emperor.com/products/azadideath-front-full-army-deal.

KolonelCrack. « The Azadi Death Front - Alternative Fierce Guardswomen ». Reddit Post. r/TheAstraMilitarum, 14 octobre 2020. https://web.archive.org/web/20220105143339/https://www.reddit.com/r/TheAstraMili tarum/comments/jbacl1/the azadi_death_front_alternative fierce/.

Kickstarter. « Azadi Death Front ». Consulté le 5 janvier 2022. https://web.archive.org/web/20201125203137/https://www.kickstarter.com/projects/4 0emperor/azadi-death-front/. $\doteq$

71. 
Par exemple : Zerocalcare. Kobane calling: facce, parole e scarabocchi da Rebibbia al confine turco siriano, 2016. http://www.worldcat.org/oclc/1140959521.

Sauloy, Mylène, et Clément Baloup. Les filles du Kurdistan: une révolution féministe, 2021. http://www.worldcat.org/oclc/1266640014.․

72. Par exemple : Marcel Cartier, Jin Jiyan Azadi, 2019 ; Marcel Cartier. Marcel Cartier - Jin Jiyan Azadi (Woman Life Freedom) [with lyrics], 2019. https://www.youtube.com/watch?v=MRinB06SzIM..

73. Dirik, Dilar. «Le slogan "Jin, jiyan, azadî” appartient aux femmes militantes, pas à Hollywood ». Kurdistan au féminin (blog), 20 octobre 2018. https://web.archive.org/web/20220105143838/https://kurdistan-aufeminin.fr/2018/10/20/le-slogan-jin-jiyan-azadi-appartient-aux-femmes-militantes-enlutte-pas-a-hollywood/. 